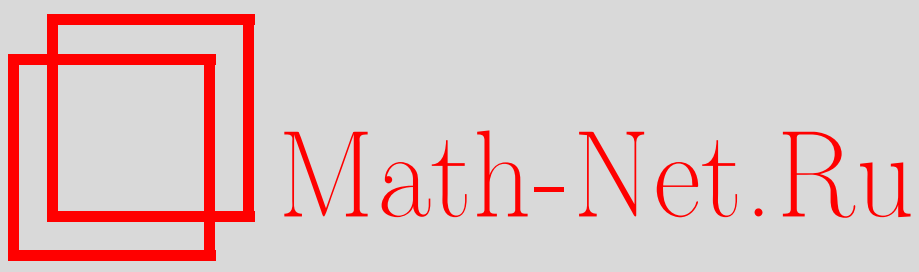

M. B. Karmanova, The area of graphs on arbitrary carnot groups with sub-lorentzian structure, Sibirsk. Mat. Zh., 2020, Volume 61, Number 4, 823-848

DOI: https://doi.org/10.33048/smzh.2020.61.408

Use of the all-Russian mathematical portal Math-Net.Ru implies that you have read and agreed to these terms of use http://www . mathnet.ru/eng/agreement

Download details:

IP: 34.239 .49 .27

April 26, 2023, 15:29:41

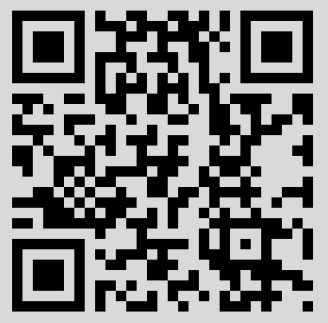


Сибирский математический журнал Июль-август, 2020. Том 61, № 4

УДК $517.518 .1+514.747$

\title{
ПЛОЩАДЬ ГРАФИКОВ НА ПРОИЗВОЛЬНЫХ ГРУППАХ КАРНО С СУБЛОРЕНЦЕВОЙ СТРУКТУРОЙ
}

\section{М. Б. Карманова}

\begin{abstract}
Аннотация. Доказана формула площади для классов отображений-графиков на группах Карно и нильпотентных градуированных группах произвольной размерности и глубины, на которых введена сублоренцева структура. Количество пространственных и временны́х направлений также произвольно.
\end{abstract}

DOI $10.33048 /$ smzh.2020.61.408

Ключевые слова: группа Карно, нильпотентная градуированная группа, многомерная сублоренцева структура, внутренний базис, внутренняя мера, формула площади.

В статье выводятся метрические свойства поверхностей-графиков на сублоренцевых структурах произвольной глубины с многомерным временем. Основой для построения таких структур является хорошо известная геометрия Минковского (см., например, [1] и цитируемые авторами литературные источники). Геометрия Минковского представляет собой структуру пространствавремени $\mathbb{R}_{1}^{n+1}$, при этом частный случай $\mathbb{R}_{1}^{4}$ является пространством событий специальной теории относительности. Ее отличительной особенностью является то, что квадрат расстояния между точками с координатами $\left(x_{1}, t_{1}\right)$ и $\left(x_{2}, t_{2}\right)$, $x_{1}, x_{2} \in \mathbb{R}^{n}, t_{1}, t_{2} \in \mathbb{R}$, вычисляется по формуле

$$
\left(x_{1}-x_{2}\right)^{2}-\left(t_{1}-t_{2}\right)^{2},
$$

т. е. вдоль временно́го направления $t$ он отрицателен, а вдоль пространственных $x \in \mathbb{R}^{n}$ положителен. Таким образом, длина вектора с ненулевыми координатами также может быть как положительной, так и отрицательной или равной нулю. Если у поверхности в $\mathbb{R}_{1}^{n+1}$ все касательные векторы имеют положительную длину, то такая поверхность называется пространственноподобной, и локально она представима в виде графика, где временна́я координата зависит от остальных: $t=\psi(x), x \in \mathbb{R}^{n}$. При определенных требованиях на гладкость определяющего поверхность отображения можно выписать уравнения, описывающие поверхность максимальной площади, из которых следует, что средняя кривизна максимальных поверхностей равна нулю. Согласно гипотезе Нильсена $[2,3]$ решения уравнения тяготения Эйнштейна физически содержательны тогда и только тогда, когда они реализуемы в виде таких поверхностей в $\mathbb{R}_{1}^{n+1}$.

Публикация выполнена при финансовой поддержке Регионального математического центра Новосибирского государственного университета.

(c) 2020 Карманова М. Б. 
Подробнее о геометрии Минковского, интерпретациях и применениях можно ознакомиться в [1] и упомянутых там источниках, например, $[2,3]$ и др.

Неголономный вариант такой геометрии, основанный на введении расстояния со свойствами (1) на субримановых структурах и называемый сублоренцевой геометрией, начали исследовать относительно недавно [4-14], как и его приложения в физике $[15,16]$. Практически в то же время, что и неголономное обобщение, стали изучаться структуры с многомерным временем [17-19], но на евклидовых пространствах. Отметим, что вопрос о вычислении площади поверхностей на вышеперечисленных аналогах до недавнего времени был открыт даже в частных случаях.

В последнее время автором установлен ряд результатов геометрической теории меры с применением к теории экстремальных поверхностей в частных случаях четырех- и пятимерных сублоренцевых структур [20-26], а также для двуступенчатых сублоренцевых структур, в том числе, с многомерным временем, имеющих произвольную размерность [27-31].

В данной работе снимается ограничение на глубину образа и прообраза отображения, по которому строится график. Кроме того, сублоренцева структура, как и понятие пространственноподобной поверхности, описывается в самом общем случае. Основной результат - формулы адаптированной площади для пространственноподобных поверхностей-графиков, построенных по классам отображений нильпотентных градуированных групп и групп Карно.

Краткий обзор результатов статьи изложен в $[32,33]$.

\section{1. Основные определения и свойства}

Для формулировки и дальнейшего решения задачи введем необходимые определения и опишем важные свойства исследуемых структур.

ОПРеДЕЛЕНИЕ 1.1 (см., например, [34]). Группой Карно называется связная односвязная стратифицированная группа Ли $\mathbb{G}$, алгебра Ли $V$ которой представляется в виде прямой суммы

$$
V=\bigoplus_{k=1}^{M} V_{i}, \quad\left[V_{1}, V_{k}\right]=V_{k+1}, k=1, \ldots, M-1, \quad\left[V_{1}, V_{M}\right]=\{0\}
$$

Число $M$ называется глубиной группы $\mathbb{G}$. Размерность $V_{k}$ в каждой точке будем обозначать символом $\operatorname{dim} V_{k}, k=1, \ldots, M$.

ОПРЕДЕЛЕНИЕ 1.2. Если в (2) условия $\left[V_{1}, V_{k}\right]=V_{k+1}$ и $\left[V_{1}, V_{M}\right]=\{0\}$ заменить условиями $\left[V_{1}, V_{k}\right] \subset V_{k+1}, k=1, \ldots, M-1$, и $\left[V_{k}, V_{M}\right]=\{0\}, k=$ $1, \ldots, M$, то $\mathbb{G}$ называется нильпотентной градуированной группой (Ли).

Из определений следует, что группа Карно является частным случаем нильпотентной градуированной группы Ли.

ОБОЗНАчЕНИЕ 1.3. ПолОЖим $n_{1}=\operatorname{dim} V_{1}$ и $n_{i}=\sum_{k=1}^{i} \operatorname{dim} V_{k}, i=2, \ldots, M$.

ОПРЕДЕЛЕНИЕ 1.4. Пусть $N$ - топологическая размерность группы $\mathbb{G}$ и $X_{1}, X_{2}, \ldots, X_{N}-$ левоинвариантные векторные поля на $\mathbb{G}$, образующие базис алгебры Ли $V$, причем

$$
\begin{cases}X_{1}, \ldots, X_{\operatorname{dim} V_{1}} & \text { - базис } V_{1}, \\ X_{n_{k-1}+1}, \ldots, X_{n_{k}} & \text { - базис } V_{k}, k=2, \ldots, M .\end{cases}
$$


Если $X_{j} \in V_{k}, k=1, \ldots, M$, то число $k$ называется степенъю поля $X_{j}$ и обозначается символом $\operatorname{deg} X_{j}, j=1, \ldots, N$. Векторные поля степени 1 далее будем называть горизонтальными.

ЗАмечАниЕ 1.5. Базис выбран таким образом, что любое базисное поле принадлежит одному из $V_{k}$ на всей группе $\mathbb{G}, k=1, \ldots, M$. Иными словами, для каждого векторного поля однозначно определено значение его степени.

ОБОЗНАчение 1.6. Обозначим символом $\mathbf{0}$ единицу группы $\mathbb{G}$.

ЗАмечАниЕ 1.7. Обозначение единицы группы нулем мотивировано тем, что многие рассуждения проводятся в нормальных координатах, в том числе относительно этой единицы (см. далее определение 1.15). Очевидно, что в этом случае координаты единицы нулевые.

ОБОЗНАчЕНИЕ 1.8. Если $x=\exp \left(\sum_{j=1}^{N} x_{j} X_{j}\right)(\mathbf{0})$, то переменные при полях степени $k$ будем называть переменными степени $k, k=1, \ldots, M$.

Для описания групповой операции понадобится следующее понятие.

ОПРЕДЕЛЕНИЕ 1.9. Для мультииндекса $\mu=\left(\mu_{1}, \ldots, \mu_{N}\right)$ его весовая норма равна

$$
|\mu|_{h}=\sum_{j=1}^{N} \mu_{j} \operatorname{dim} V_{j}
$$

Из формулы Бейкера - Кэмпбелла - Хаусдорфа и условия левоинвариантности базисных полей выводятся следующие выражения для групповой операции на $\mathbb{G}$. Если

$$
x=\exp \left(\sum_{j=1}^{N} x_{j} X_{j}\right)(\mathbf{0}), \quad y=\exp \left(\sum_{j=1}^{N} y_{j} X_{j}\right)(\mathbf{0}),
$$

то

$$
x \cdot y=z=\exp \left(\sum_{j=1}^{N} z_{j} X_{j}\right)(\mathbf{0})
$$

где

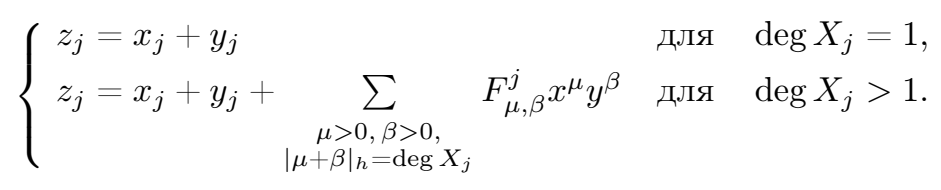

Геометрическая интерпретация умножения $x \cdot y$ состоит в следующем. Сначала движение идет вдоль интегральной линии векторного поля $\sum_{j=1}^{N} x_{j} X_{j}$ с началом в 0 до точки $x$, а затем - вдоль интегральной линии векторного поля $\sum_{j=1}^{N} y_{j} X_{j}$ с началом в $x$. Таким образом, интегральная линия поля $\sum_{j=1}^{N} z_{j} X_{j}$ соединяет точки $\mathbf{0}$ и $z=\exp \left(\sum_{j=1}^{N} y_{j} X_{j}\right)(x)$.

ОПРЕдЕЛЕниЕ 1.10. Константы $\left\{F_{\mu, \beta}^{j}\right\}_{j, \mu, \beta}$ называются структурными константами группы $\mathbb{G}$.

На группах Карно часто используется метрика Карно - Каратеодори $d_{c c}$, согласованная с неголономной структурой (см., например, [35]). Однако до сих 
пор остаются открытыми вопросы о строении ее шаров. Кроме того, ее не всегда возможно использовать на нильпотентных градуированных группах, поэтому в таких случаях применяется квазиметрика $d_{\infty}$, которая на группах Карно локально билипшицево эквивалентна $d_{c c}$ : если $w=\exp \left(\sum_{i=1}^{N} w_{i} X_{i}\right)(v)$, то

$$
d_{\infty}(v, w)=\max _{i=1, \ldots, N}\left\{\left|w_{i}\right|^{\frac{1}{\operatorname{deg} X_{i}}}\right\}
$$

Мы же будем использовать следующую величину, локально билипшицево эквивалентную $d_{\infty}$.

ОПРЕДЕЛЕНиЕ 1.11. Пусть $\mathbb{G}$ - нильпотентная градуированная группа топологической размерности $N$ и $w=\exp \left(\sum_{i=1}^{N} w_{i} X_{i}\right)(v)$. Определим величину $d_{2}$ следующим образом:

$$
d_{2}(v, w)=\max _{k=1, \ldots, M}\left\{\left(\sum_{j: \operatorname{deg} X_{j}=k} w_{j}^{2}\right)^{\frac{1}{2 k}}\right\}
$$

Свойство 1.12. Величина $d_{2}(v, w)$ локально является квазиметрикой: она неотрицательна (и равна нулю тогда и только тогда, когда $v=w)$, обладает свойством симметричности и для всякой окрестности $D \subset \mathbb{G}$ существует константа $C_{D}<\infty$ такая, что обобщенное неравенство треугольника

$$
d_{2}(v, w) \leq C_{D}\left(d_{2}(v, u)+d_{2}(u, w)\right)
$$

верно для всех $v, u, w \in D$.

ОБОЗНАЧЕНИЕ 1.13. Шар в квазиметрике $d_{2}$ радиуса $r$ с центром в точке $v$, равный $\left\{w \in \mathbb{G}: d_{2}(v, w)<r\right\}$, обозначим символом $\operatorname{Box}_{2}(v, r)$.

Свойство 1.14. Рассмотрим точку $v \in \mathbb{G} и\left(w_{1}, \ldots, w_{N}\right) \in \mathbb{R}^{N}$. Определим отображение $\theta_{v}: \mathbb{R}^{N} \rightarrow \mathbb{G}$ следующим образом:

$$
\theta_{v}\left(w_{1}, \ldots, w_{N}\right)=\exp \left(\sum_{i=1}^{N} w_{i} X_{i}\right)(v)
$$

Известно, что $\theta_{v}$ - гладкий диффеоморфизм.

ОПРЕДЕЛЕНИЕ 1.15. Набор $\left\{w_{i}\right\}_{i=1}^{N}$ называется нормалъными координатами или координатами первого рода (относительно $v \in \mathbb{G}$ ) точки $w=\theta_{v}\left(w_{1}, \ldots\right.$, $\left.w_{N}\right)$.

Свойство 1.16. Образ шара $\operatorname{Box}_{2}(v, r)$ при отображении $\theta_{v}^{-1}-$ декартово произведение шаров, диаметры которых равны $2 r, 2 r^{2}, \ldots, 2 r^{M}$.

Свойство 1.17. С помощью свойства 1.16 непосредственно проверяется, что хаусдорфова размерность группы $\mathbb{G}$ относительно $d_{2}$ равна

$$
\nu=\sum_{i=1}^{M} k \operatorname{dim} V_{k}
$$

Опишем классы исследуемых в статье отображений. 
Предположение 1.18. Будем рассматривать $\varphi: \Omega \rightarrow \widetilde{\mathbb{G}}$, где

(1) $\Omega \subset \mathbb{G}$ - открытое множество;

$(2) \mathbb{G}, \widetilde{\mathbb{G}} \subset \mathbb{U}$, где $\mathbb{U}-$ нильпотентная градуированная группа топологической размерности $N+\widetilde{N}$ с базисными полями $X_{1}, \ldots, X_{N}$ и $\widetilde{X}_{1}, \ldots, \widetilde{X}_{\widetilde{N}}$, алгеброй Ли векторных полей $\widehat{V}=\bigoplus_{k=1}^{\widehat{M}} \widehat{V}_{k}$, где поля $X_{1}, \ldots, X_{n_{1}}, \widetilde{X}_{1}, \ldots, \widetilde{X}_{\tilde{n}_{1}}$ составляют базис $\widehat{V}_{1}$, а $X_{n_{k-1}+1}, \ldots, X_{n_{k}}, \widetilde{X}_{\tilde{n}_{k-1}+1}, \ldots, \widetilde{X}_{\tilde{n}_{k}}-$ базис $\widehat{V}_{k}, k=2, \ldots, \widehat{M}$, единицей $\widehat{\mathbf{0}}$ и квазиметрикой $\hat{d}_{2}$;

(3) $\mathbb{G}$ - нильпотентная градуированная группа топологической размерности $N$ с базисными полями $X_{1}, \ldots, X_{N}$, алгеброй Ли векторных полей $V=$ $\bigoplus_{k=1}^{M} V_{k}$, где поля $X_{1}, \ldots, X_{n_{1}}$ составляют базис $V_{1}$, а $X_{n_{k-1}+1}, \ldots, X_{n_{k}}-$ базис $V_{k}, k=2, \ldots, M$, единицей $\mathbf{0}$ и квазиметрикой $d_{2}$;

(4) $\widetilde{\mathbb{G}}$ - нильпотентная градуированная группа топологической размерности $\widetilde{N}$ с базисными полями $\widetilde{X}_{1}, \ldots, \widetilde{X}_{\widetilde{N}}$, алгеброй Ли векторных полей $\widetilde{V}=$ $\bigoplus_{k=1}^{\widetilde{M}} \widetilde{V}_{k}$, где поля $\widetilde{X}_{1}, \ldots, \widetilde{X}_{\tilde{n}_{1}}$ составляют базис $\widetilde{V}_{1}$, а $\widetilde{X}_{\tilde{n}_{k-1}+1}, \ldots, \widetilde{X}_{\tilde{n}_{k}}-$ базис $\widetilde{V}_{k}, k=2, \ldots, \widetilde{M}$, единицей $\widetilde{\mathbf{0}}$ и квазиметрикой $\tilde{d}_{2}$;

(5) $\widehat{\mathbf{0}}=(\mathbf{0}, \widetilde{\mathbf{0}})$ и $\widehat{M}=\max \{M, \widetilde{M}\}$;

(6) каждая координатная функция $\varphi_{i}$ имеет гладкость $\left[\frac{\widehat{M}+p-1}{p}\right]$ по переменным степени $p, p=1, \ldots, M$. Здесь $\left(\varphi_{1}(x), \ldots, \varphi_{\widetilde{N}}(x)\right)=\theta_{\widetilde{\mathbf{0}}}^{-1}(\varphi(x)), x \in \Omega$.

ЗАмечАниЕ 1.19. Группы $\mathbb{G}$ и $\widetilde{\mathbb{G}}-$ подмногообразия $\mathbb{U}$, которые пересекаются по их единицам. Это пересечение совпадает с единицей $\widehat{\mathbf{0}}$ группы $\mathbb{U}$.

ЗАмечАние 1.20. Мы не накладываем строгих условий на соотношение $M$ и $\widetilde{M} ;$ см. подробности в теоремах $2.16,2.18,2.24$ и их доказательствах, а также свойство 2.22. Единственное ограничение состоит в том, что $\widetilde{M}$ не может быть строго больше $M$.

ЗАмечание 1.21. Требование п. (6) остается корректным, если точку $\widetilde{\mathbf{0}}$ заменить произвольной точкой; см. подробнее в [36].

ОПредЕлЕниЕ 1.22 . Пусть $\mathbb{G}, \widetilde{\mathbb{G}}$ и $\mathbb{U}-$ нильпотентные градуированные группы Ли, удовлетворяющие условиям предположения $1.18, \Omega \subset \mathbb{G}$ и $\varphi: \Omega \rightarrow$ $\widetilde{\mathbb{G}}$. Отображение-график $\varphi_{\Gamma}: \Omega \rightarrow \mathbb{U}$ сопоставляет точке $x$ элемент

$$
\mathbb{U} \ni \varphi_{\Gamma}(x)=\exp \left(\sum_{j=1}^{\widetilde{N}} \varphi_{j}(x) \tilde{X}_{j}\right)(x),
$$

где

$$
\exp \left(\sum_{j=1}^{\widetilde{N}} \varphi_{j}(x) \widetilde{X}_{j}\right)(\widetilde{\mathbf{0}})=\varphi(x) \in \widetilde{\mathbb{G}} .
$$

Опишем специфику отображений-графиков, а именно связь их свойств со свойствами отображений, по которым они строятся. Приведенные результаты будут использованы далее при вычислении локального искажения меры и доказательства формулы площади. 
ОПРЕдЕлЕниЕ 1.23. Пусть $\mathbb{G}, \widetilde{\mathbb{G}}-$ нильпотентные градуированные группы, $\Omega \subset \mathbb{G}$ и $\varphi: \Omega \rightarrow \widetilde{\mathbb{G}}$. Отображение $\varphi$ называется липшицевым во внутреннем смысле, или липшицевым в субримановом смысле, если

$$
\tilde{d}_{2}(\varphi(y), \varphi(x)) \leq L d_{2}(y, x)
$$

для $L<\infty$ и всех $x, y \in \Omega$.

В силу формул (3) легко видеть, что отображения-графики в общем случае не являются липшицевыми в субримановом смысле, если таковым является исходное отображение (в отличие от классического случая; см., например, частный случай в [37]). Отображения-графики гёльдеровы с показателем $1 / \widehat{M}$ :

$$
\hat{d}_{2}\left(\varphi_{\Gamma}(y), \varphi_{\Gamma}(x)\right) \leq K d_{2}(x, y)^{1 / \widehat{M}}, \quad 0<K<\infty .
$$

Несмотря на то, что разработанная для липшицевых отображений теория субримановой дифференцируемости неприменима к отображениям-графикам, существуют способы описать их метрические свойства через дифференциальные характеристики исходных отображений (см. ниже теорему 1.32). Для детального описания напомним основные определения и результаты теории.

ОПРЕдЕЛЕниЕ 1.24 ([35], см. также [38]). Пусть $\mathbb{G}$ и $\widetilde{\mathbb{G}}-$ нильпотентные градуированные группы, $\Omega \subset \mathbb{G}$ и $\varphi: \Omega \rightarrow \widetilde{\mathbb{G}}$. Отображение $\varphi$ является $h c$ дифферениируемым, или дифферениируемым в субримановом смысле, в (предельной) точке $x \in \Omega$, если существует горизонтальный гомоморфизм $\mathscr{L}_{x}:$ $\mathbb{G} \rightarrow \widetilde{\mathbb{G}}$ такой, что

$$
\widetilde{d}_{2}\left(\varphi(y), \mathscr{L}_{x}\langle y\rangle\right)=o\left(d_{2}(x, y)\right) \quad \text { при } \Omega \ni y \rightarrow x .
$$

$h c$-Дифференииал (или субриманов дифференциал) $\mathscr{L}_{x}$ обозначается символом $\widehat{D} \varphi(x)$.

Теорема 1.25 ([35], см. также [38] для измеримых множеств). Пусть $\mathbb{G}-$ группа Карно, $\Omega \subset \mathbb{G}$ - открытое множество, $\widetilde{\mathbb{G}}-$ нильпотентная градуированная группа и $\varphi: \Omega \rightarrow \widetilde{\mathbb{G}}-$ липшицево во внутреннем смысле отображение. Тогда $\varphi$ является $h c$-дифференцируемым почти всюду.

Важным подклассом липшицевых в субримановом смысле отображений являются контактные отображения класса $C_{H}^{1}$.

ОПРЕДЕлЕНИЕ 1.26. Отображение $\varphi$ нильпотентных градуированных групп является контактным отображением класса $C_{H}^{1}$, если его горизонтальные производные существуют и непрерывны, а образы горизонтальных полей принадлежат горизонтальному подрасслоению образа.

Известно [38], что такие отображения, определенные на открытых множествах $\Omega \subset \mathbb{G}$ групп Карно, непрерывно $h c$-дифференцируемы всюду.

ЗАмечАниЕ 1.27. Случай определенного на группе Карно контактного отображения $\varphi$ для задачи, решаемой в статье, является основным. О распространении результатов на классы неконтактных отображений нильпотентных градуированных групп говорится отдельно; см. теорему 2.18 .

Следующее понятие является основным инструментом работы с отображениями-графиками и другими классами гёльдеровых в субримановом смысле отображений неголономных структур. Оно является обобщением субримановой дифференцируемости. 
ОПРЕдЕЛЕниЕ 1.28 (см., например, [36]). Пусть $\mathbb{G}$ и $\widetilde{\mathbb{G}}-$ нильпотентные градуированные группы Ли, $\Omega \subset \mathbb{G}, \psi: \Omega \rightarrow \widetilde{\mathbb{G}}$ и функция $\tilde{\mathfrak{d}}: \psi(\Omega) \times \widetilde{\mathbb{G}} \rightarrow \mathbb{R}_{+}$ такова, что $\tilde{\mathfrak{d}}(x, x)=0$ и $\tilde{\mathfrak{d}}(x, y)=\tilde{\mathfrak{d}}(y, x)$ для всех точек ее области определения. Будем говорить, что $\psi$ полиномиально субриманово дифференцируемо, или по-

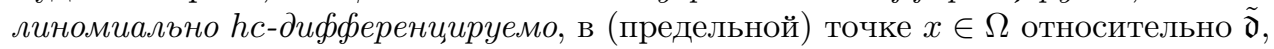
если существует отображение $\mathscr{L}_{x}: \mathbb{G} \rightarrow \widetilde{\mathbb{G}}$ такое, что

1) $\tilde{\mathfrak{d}}\left(\psi(w), \mathscr{L}_{x}\langle w\rangle\right)=o\left(d_{2}(x, w)\right), \Omega \ni w \rightarrow x$

2) $\mathscr{L}_{x}(w)=\theta_{\psi(x)} \circ L_{x} \circ \theta_{x}^{-1}(w)$, где компоненты $L_{x}$ - полиномы по $w_{1}, \ldots, w_{N}$, $\mathrm{a} \exp \left(\sum_{j=1}^{N} w_{j} X_{j}\right)(x)=w$.

Отображение $\mathscr{L}_{x}$ называется полиномиальным субримановым дифференциалом, или полиномиальным $h c$-дифференциалом, отображения $\psi$ в точке $x$.

ОБоЗНАчЕНИЕ 1.29. Здесь и далее полиномиальный субриманов дифференциал $\mathscr{L}_{x}$ отображения $\psi$ в точке $x$ будем обозначать символом $\widehat{D}_{P} \psi(x)$.

ЗАмечание 1.30. Требуемые в определении 1.28 свойства не обеспечивают

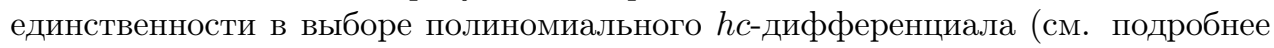
в [36]). По умолчанию будем рассматривать минимально возможный приближающий отображение полином. При необходимости более точной аппроксимации вид полиномиального $h c$-дифференциала оговорен специально.

ОБозНАчениЕ 1.31. Далее в статье символ $(\varphi(x))^{\eta}$ обозначает произведение $\prod_{j=1}^{\widetilde{N}} \varphi_{j}(x)^{\eta_{j}}$ для набора $\left\{\varphi_{j}(x)\right\}_{j=1}^{\widetilde{N}}$ из определения 1.22 и мультииндекса $\eta=\left(\eta_{1}, \ldots, \eta_{\widetilde{N}}\right)$, а символ $(\widehat{D} \varphi(x)\langle y\rangle)^{\tau}-$ произведение $\prod_{j=1}^{\widetilde{N}}(\widehat{D} \varphi(x)\langle y\rangle)_{j}^{\tau_{j}}$ для мультииндекса $\tau=\left(\tau_{1}, \ldots, \tau_{\widetilde{N}}\right)$, где $\left((\widehat{D} \varphi(x)\langle y\rangle)_{1}, \ldots,(\widehat{D} \varphi(x)\langle y\rangle)_{\widetilde{N}}\right)-$ координаты $\widehat{D} \varphi(x)\langle y\rangle$ относительно $\varphi(x)$ в базисе $\left\{\widetilde{X}_{j}\right\}_{j=1}$.

Теорема $1.32[36]$. Пусть $\varphi \in C_{H}^{1}(\Omega, \widetilde{\mathbb{G}})$. Отображение-график $\varphi_{\Gamma}: \Omega \rightarrow \mathbb{U}$ полиномиально субриманово дифференцируемо всюду, и $\widehat{D}_{P} \varphi_{\Gamma}(x)$ сопоставляет элементу $\exp \left(\sum_{p=1}^{N} y_{p} X_{p}\right)(x)$ в координатах относительно $\varphi_{\Gamma}(x)$ набор $\left(\Delta_{1}(x, y)\right.$, $\left.\ldots, \Delta_{N}(x, y), \widetilde{\Delta}_{1}(x, y), \ldots, \widetilde{\Delta}_{\widetilde{N}}(x, y)\right)$, где

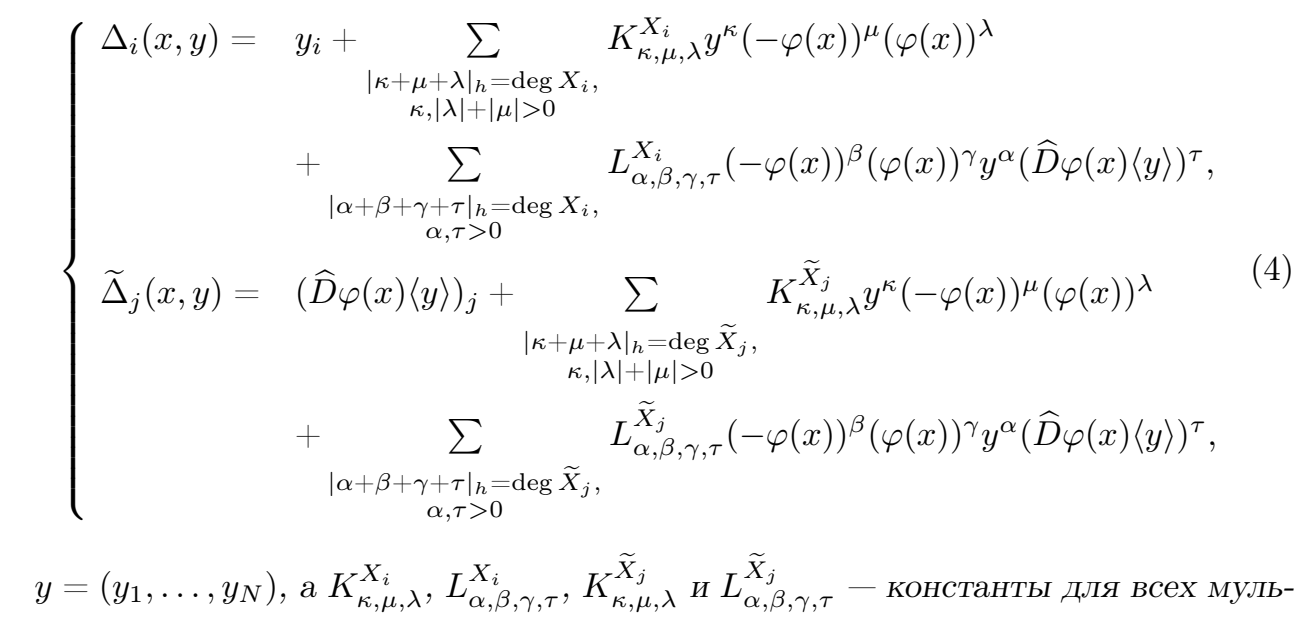


тииндексов $\kappa, \mu, \lambda, \alpha, \beta, \gamma, \tau, i=1, \ldots, N, j=1, \ldots, \tilde{N}$.

ЗАмЕчАНИЕ 1.33. Подчеркнем, что полиномиальный субриманов дифференциал зависит от фиксированной точки $x$ и от точек $y$ из ее окрестности.

ОБОЗНАЧЕНИЕ 1.34. Действие полиномиального субриманова дифференциала отображения $\psi$ в точке $x$ на элементе $y$ будем обозначать символом $\widehat{D}_{P} \psi(x)\langle y\rangle$.

Из установленных в теореме 1.32 выражений координат полиномиального субриманова дифференциала видно, что неголономные структуры образа и прообраза не являются согласованными, т. е. координаты при каждом базисном поле $X$ не всегда сравнимы с величиной $O\left(d_{\infty}(x, y)^{\operatorname{deg} X}\right)$. Чтобы такое условие локально выполнялось, необходимо изменение базиса в образе в окрестности $\varphi_{\Gamma}(x)$. Введем понятие адаптированного базиса и сформулируем результаты.

ОПрЕДЕЛЕниЕ 1.35 [36]. Пусть $\mathbb{G}$ и $\widetilde{G}-$ нильпотентные градуированные группы, $\Omega \subset \mathbb{G}$ и $\psi: \Omega \rightarrow \widetilde{\mathbb{G}}$ полиномиально $h c$-дифференцируемо. Если координаты $\left\{\kappa_{j}\right\}_{j=1}^{\widetilde{N}}$ полиномиального субриманова дифференциала $\widehat{D}_{P} \psi(x)\langle y\rangle$, рассмотренные относительно точки $\psi(x)$ в базисе $\left\{Y_{i}\right\}_{i=1}^{\widetilde{N}}$, обладают свойством $\left|\kappa_{j}\right|=$ $O\left(d_{\infty}(x, y)^{\operatorname{deg} \widetilde{X}_{j}}\right)$ для всех $y$ из некоторой окрестности точки $x, j=1, \ldots, \widetilde{N}$, то базис $\left\{Y_{i}\right\}_{i=1}^{\widetilde{N}}$ называется внутренним, или адаптированным (в точке $x$ ).

ОБОЗНАЧЕНИЕ 1.36. Рассмотрим следующую матрицу:

$$
\left(\begin{array}{cccccc}
E_{\operatorname{dim} V_{1}} & 0 & 0 & \ldots & 0 & 0 \\
0 & E_{\operatorname{dim} V_{2}} & 0 & \ldots & 0 & 0 \\
0 & 0 & E_{\operatorname{dim} V_{3}} & \ldots & 0 & 0 \\
\ldots & & & \ldots & 0 & 0 \\
0 & 0 & 0 & \ldots & 0 & E_{\operatorname{dim} V_{M}} \\
(\widehat{D} \varphi)_{\widetilde{V}_{1}, V_{1}}(x) & 0 & 0 & \ldots & 0 & 0 \\
0 & (\widehat{D} \varphi)_{\widetilde{V}_{2}, V_{2}}(x) & 0 & \ldots & 0 & 0 \\
0 & 0 & (\widehat{D} \varphi)_{\widetilde{V}_{3}, V_{3}}(x) & \ldots & 0 & 0 \\
0 & 0 & 0 & \ldots & 0 & (\widehat{D} \varphi)_{\widetilde{V}_{M}, V_{M}}(x)
\end{array}\right),
$$

где $E_{\operatorname{dim} V_{l}}-$ единичные матрицы размера $\operatorname{dim} V_{l}, l=1, \ldots, M, \mathrm{a}\left(\widehat{D}_{\varphi}\right)_{\widetilde{V}_{j}, V_{j}}(x)$, $j=1, \ldots, \min \{M, \widetilde{M}\}$, - диагональные блоки матрицы $h c$-дифференциала отображения $\varphi$, и $(\widehat{D} \varphi)_{\widetilde{V}_{M}, V_{M}}(x)=\varnothing$, если $\widetilde{M}<M$. Если $\widetilde{M}>M$, то в матрице $(5)$ присутствуют дополнительные нулевые строки.

Оператор, полученный из дифференциала $D\left(\widehat{D}_{P} \varphi_{\Gamma}(x)\right)(x)$, матрица которого совпадает с матрицей (5) (без изменения базиса), будем обозначать символом $D_{\text {diag }}\left(\widehat{D}_{P} \varphi_{\Gamma}(x)\right)(x)$.

Теорема 1.37 [36]. Пусть $\varphi$ в условиях предположения 1.18 является контактным отображением, a $\mathbb{G}$ - группа Карно. Тогда для всех $x \in \Omega$ существует адаптированный базис $\left\{{ }^{x} X_{1}, \ldots,{ }^{x} X_{N},{ }^{x} \widetilde{X}_{1}, \ldots,{ }^{x} \widetilde{X}_{\widetilde{N}}\right\}$ такой, что множество

$$
\left({ }^{x} \tilde{\theta}_{\varphi_{\Gamma}(x)} \circ \theta_{\varphi_{\Gamma}(x)}^{-1}\right)\left(D_{\operatorname{diag}}\left(\widehat{D}_{P} \varphi_{\Gamma}(x)\right)(x)\langle\operatorname{Box}(x, r)\rangle\right)
$$

совпадает с $\widehat{D}_{P} \varphi_{\Gamma}(x)\langle\operatorname{Box}(x, r)\rangle$. Здесь $\theta_{\varphi_{\Gamma}(x)}$ - отображение нормальных координат в исходном базисе, $\mathrm{a}^{x} \tilde{\theta}_{\varphi_{\Gamma}(x)}-$ в адаптированном базисе. 
Справедливо следующее утверждение для рассматриваемых классов гёльдеровых отображений.

Теорема 1.38 [36]. Если $\varphi$ не обладает свойством контактности, а все остальные условия предположения 1.18 выполнены, то справедливы результаты теорем 1.32 и 1.37 с заменой координат $\widehat{D} \varphi(x)\langle y\rangle$ координатами $\widehat{D}_{P} \varphi(x)\langle y\rangle$. $B$ частности, в матрице (5) блоки $(\widehat{D} \varphi)_{\widetilde{V}_{k}, V_{k}}(x)$ заменяются на $D\left(\widehat{D}_{P} \varphi\right)_{\widetilde{V}_{k}, V_{k}}(x)$, $k=1, \ldots, \min \{M, \widetilde{M}\}$. Здесь $\widehat{D}_{P} \varphi(x)$ приближает $\varphi$ в точке у с точностью до о $\left(d_{2}(x, y)^{\widehat{M}}\right)$.

ОБОЗНАчЕНИЕ 1.39. Если $w=\exp \left(\sum_{i=1}^{N} w_{i}^{x} X_{i}+\sum_{j=1}^{\widetilde{N}} w_{N+j}{ }^{x} \widetilde{X}_{j}\right)(v)$, то

$$
{ }^{x} \hat{d}_{2}(v, w)=\max _{k=1, \ldots, \max \{M, \widetilde{M}\}}\left\{\left(\sum_{i: \operatorname{deg} X_{i}=k} w_{j}^{2}+\sum_{j: \operatorname{deg} \widetilde{X}_{j}=k} w_{N+j}^{2}\right)^{\frac{1}{2 k}}\right\} .
$$

\section{2. Сублоренцевы структуры и мера на поверхностях}

Опишем сублоренцеву структуру на $\mathbb{U}$. Так как на таких структурах существуют направления, квадрат длины вдоль которых отрицателен, разобьем базисные векторные поля в каждом $\widehat{V}_{k}$ по этому признаку на «положительные» и «отрицательные», $k=1, \ldots, \widehat{M}$.

ОПИСАНИЕ 2.1. Положим

$$
\left\{X_{1}, \ldots, X_{N}, \widetilde{X}_{1}, \ldots, \widetilde{X}_{\widetilde{N}}\right\}=\left\{Y_{1}, \ldots, Y_{\widehat{N}}\right\}
$$

где $\widehat{N}=N+\widetilde{N}$. Далее, пусть

$$
\widehat{V}_{k}=\operatorname{span}\left\{\widehat{V}_{k}^{+}, \widehat{V}_{k}^{-}\right\}, \quad \text { где } \widehat{V}_{k}^{+} \cap \widehat{V}_{k}^{-}=\{0\} \text { и } \widehat{V}_{k}^{-} \subseteq \widetilde{V}_{k}, k=1, \ldots, \widehat{M},
$$

причем каждое базисное поле из $\widehat{V}_{k}$ принадлежит либо $\widehat{V}_{k}^{+}$, либо $\widehat{V}_{k}^{-}, k=$ $1, \ldots, \widehat{M}$.

Приведем определения сублоренцевых расстояния и меры сразу для внутреннего базиса, так как они будут использоваться только в этом виде. Для исходного базиса все определения аналогичны (см. также [30]).

ОБОЗНАчениЕ 2.2. Для каждого фиксированного $x \in \Omega$ положим $\left\{{ }^{x} Y_{1}, \ldots\right.$, $\left.{ }^{x} Y_{\widehat{N}}\right\}=\left\{{ }^{x} X_{1}, \ldots,{ }^{x} X_{N},{ }^{x} \widetilde{X}_{1}, \ldots,{ }^{x} \widetilde{X}_{\widetilde{N}}\right\}$. Здесь каждое адаптированное поле ${ }^{x} Y_{k}$ заменяет соответствующее базисное поле $Y_{k}, k=1, \ldots, \widehat{N}$.

ОПРедЕлЕниЕ 2.3. Фиксируем $x \in \Omega$. Для векторного поля $T=\sum_{j=1}^{\widehat{N}} y_{j}{ }^{x} Y_{j}$ с постоянными коэффициентами положим квадратную сублоренцеву норму равной

$$
{ }^{x} \mathbf{d}_{2}^{S L^{2}}(T)=\max _{k=1, \ldots, \widehat{M}}\left\{\operatorname{sgn}\left(\sum_{j: Y_{j} \in \widehat{V}_{k}^{+}} y_{j}^{2}-\sum_{j: Y_{j} \in \widehat{V}_{k}^{-}} y_{j}^{2}\right) \cdot\left|\sum_{j: Y_{j} \in \widehat{V}_{k}^{+}} y_{j}^{2}-\sum_{j: Y_{j} \in \widehat{V}_{k}^{-}} y_{j}^{2}\right|^{1 / k}\right\} .
$$


Квадратная сублоренцева норма ${ }^{x} \mathbf{d}_{2}^{S L^{2}}\left(T\left(x_{0}\right)\right)$ вектора, равного

$$
T\left(x_{0}\right)=\sum_{j=1}^{\widehat{N}} y_{j}^{x} Y_{j}\left(x_{0}\right)
$$

определяется аналогично.

ОПРЕДЕЛЕНИЕ 2.4. Пусть $w=\exp (T)(v)$, где $T=\sum_{j=1}^{\widehat{N}} y_{j}^{x} Y_{j}$. Положим ${ }^{x} \mathfrak{d}_{2}^{2}(v, w)={ }^{x} \mathbf{d}_{2}^{S L^{2}}(T)$. Величину ${ }^{x} \mathfrak{d}_{2}^{2}(v, w)$ будем называть квадратным сублоренцевым расстоянием.

ОПРЕДЕЛЕНИЕ 2.5. Шар относительно ${ }^{x} \mathfrak{d}_{2}^{2}$ с центром в точке $v$ радиуса $r$ это множество

$$
{ }^{x} \operatorname{Box}_{2}^{\mathfrak{d}}(v, r)=\left\{w \in \mathbb{U}:{ }^{x} \mathfrak{d}_{2}^{2}(v, w)<r^{2}\right\} .
$$

ЗАмЕчАниЕ 2.6. Для исследования метрических свойств поверхностей на сублоренцевых структурах будет достаточно квадратной сублоренцевой нормы и квадратного сублоренцева расстояния (см. подробности в [30]).

Здесь и далее в статье перегруппируем поля на $\mathbb{U}$ по степеням.

ЗАмЕчАниЕ 2.7. При отображении $\theta_{v}^{-1}$ шар $^{x} \operatorname{Box}_{2}^{\mathfrak{d}}(v, r)$ переходит в декартово произведение множеств

$$
\begin{aligned}
& \left\{\left(y_{n_{k-1}+\tilde{n}_{k-1}+1}, \ldots, y_{n_{k}+\tilde{n}_{k}}\right):\right. \\
& \left.\operatorname{sgn}\left(\sum_{j: Y_{j} \in \widehat{V}_{k}^{+}} y_{j}^{2}-\sum_{j: Y_{j} \in \widehat{V}_{k}^{-}} y_{j}^{2}\right) \cdot\left|\sum_{j: Y_{j} \in \widehat{V}_{k}^{+}} y_{j}^{2}-\sum_{j: Y_{j} \in \widehat{V}_{k}^{-}} y_{j}^{2}\right|<r^{2 k}\right\},
\end{aligned}
$$

$k=1, \ldots, \widehat{M}, n_{0}=\tilde{n}_{0}=0$. Здесь мы предполагаем, что координаты $\left(y_{1}, \ldots, y_{\widehat{N}}\right)$ упорядочены по степеням соответствующих векторных полей. Иными словами, координаты $\left(y_{n_{k-1}+\tilde{n}_{k-1}+1}, \ldots, y_{n_{k}+\tilde{n}_{k}}\right)$ соответствуют полям из $\widehat{V}_{k}$.

В геометрии Минковского для теории максимальных поверхностей интерес представляют пространственноподобные поверхности, т. е. те, касательные векторы к которым имеют положительную длину [1]. В [30] сформулировано обобщение такого понятия и установлено соответствующее свойство для широких классов поверхностей на двуступенчатых сублоренцевых структурах. Дело в том, что у классов поверхностей-графиков (см., например, [29, 30]) касательных векторов может не существовать на множестве положительной меры. Поэтому определение пространственноподобия сформулировано в терминах светового конуса: локально поверхность должна лежать вне любого светового конуса с вершиной на этой поверхности. В классическом случае оба понятия совпадают. Сформулируем признак пространственноподобия таким же образом, как в [30].

ОПРЕДЕЛЕНИЕ 2.8. Пусть $\psi: \Omega \rightarrow \mathbb{U}, \Omega \subset \mathbb{G}$, биективно на свой образ, $S=\psi(\Omega)$ и $v \in S$. Множество

$$
\left\{\exp \left(\sum_{j=1}^{\widehat{N}} y_{j}{ }^{-1}(v) Y_{j}\right)(v): \sum_{j: Y_{j} \in \widehat{V}_{k}^{+}} y_{j}^{2}-\sum_{j: Y_{j} \in \widehat{V}_{k}^{-}} y_{j}^{2}=0, k=1, \ldots, \widehat{M}\right\}
$$

называется световым конусом с центром в $v$. 
ОПРЕДЕЛЕНИЕ 2.9 (cp. [23]). Поверхность-образ $S \subset \mathbb{U}$ биективного на свой образ отображения $\psi: \Omega \rightarrow \mathbb{U}, \Omega \subset \mathbb{G}$, называется пространственноподобной (относительно адаптированного базиса), если для любой ее точки $v \in S$ существует $r_{0}>0$ такое, что пересечение $S \cap B(v, r)$ (за исключением $v$ ) лежит строго вне светового конуса с центром в $v$ для любого $r \leq r_{0}$, т. е. в множестве

$$
\left\{\exp \left(\sum_{j=1}^{\widehat{N}} y_{j}^{\psi^{-1}(v)} Y_{j}\right)(v): \max _{k=1, \ldots, \widehat{M}}\left\{\sum_{j: Y_{j} \in \widehat{V}_{k}^{+}} y_{j}^{2}-\sum_{j: Y_{j} \in \widehat{V}_{k}^{-}} y_{j}^{2}\right\}>0\right\} .
$$

Здесь $B-$ шар в (суб)римановой (квази)метрике.

Для рассматриваемых в статье отображений, включая принадлежащие классу $C_{H}^{1}$, отображение, обратное к графику, также будет непрерывным. Чтобы в этом убедиться, достаточно повторить рассуждения, используемые для вывода (4) в [36], для обратного с учетом того, что координаты, соответствующие полям $\widetilde{X}_{1}, \ldots, \widetilde{X}_{\widetilde{N}}$, должны быть нулевыми. Отсюда выводится зависимость компонент $\theta_{\varphi(x)}^{-1}(\varphi(y))$ от набора $\theta_{\varphi_{\Gamma}(x)}^{-1}\left(\varphi_{\Gamma}(y)\right)$, а также оценки, доказывающие непрерывность $\varphi_{\Gamma}^{-1}$. Поэтому при проверке свойства пространственноподобия достаточно рассматривать либо $S \cap B(v, r)$, либо $\varphi_{\Gamma}\left(U\left(\varphi_{\Gamma}^{-1}(v)\right)\right)$, где $U\left(\varphi_{\Gamma}^{-1}(v)\right)$ - некоторая окрестность $\varphi_{\Gamma}^{-1}(v)$.

Введем для пространственноподобных поверхностей-графиков следующую меру.

ОПРЕДЕЛЕНИЕ 2.10. Фиксируем точку $p \in \Omega$ и ее окрестность $\mathscr{U} \Subset \Omega$, $\mathscr{U} \ni p$, на которой величина $o(1)$ из определения $h c$-дифференцируемости достаточно мала, и рассмотрим $\delta_{0}>0$. Пусть $S \subset \varphi_{\Gamma}(\mathscr{U})$ и $\delta \in\left(0, \delta_{0}\right)$. Положим внутреннюю, или адаптированную, меру множества $A \subset S$ равной

$S L \mathscr{H}_{\Gamma}^{\nu}(A)=\prod_{j=1}^{M} \omega_{\operatorname{dim} V_{j}} \liminf _{\delta \rightarrow 0}\left\{\sum_{i \in \mathbb{N}} r_{i}^{\nu}: \bigcup_{i \in \mathbb{N}}^{\varphi_{\Gamma}^{-1}\left(x_{i}\right)} \operatorname{Box}_{2}^{\mathfrak{d}}\left(x_{i}, r_{i}\right) \supset A, x_{i} \in A, r_{i}<\delta\right\}$.

Глобально такая мера вводится аналогично работе [30] и др.: область определения исчерпывается компактными подмножествами, каждое из них покрывается конечным числом окрестностей, обладающих свойствами $\mathscr{U}$, таким образом, что найдется $r_{0}>0$, что на любом шаре меньшего радиуса $o(1)$ из определения $h c$-дифференцируемости не будет превосходить некоторой фиксированной величины, для всякого такого покрытия выбирается достаточно малое $\delta_{0}$ (определяемое величиной $r_{0}$ ), а покрытие $A \subset \varphi_{\Gamma}(\Omega)$ формируется из множеств $\left\{\varphi_{\Gamma}^{-1}\left(x_{i}\right) \operatorname{Box}_{2}^{\mathfrak{d}}\left(x_{i}, r_{i}\right) \cap \varphi_{\Gamma}\left(\mathscr{U}\left(\varphi_{\Gamma}^{-1}\left(x_{i}\right)\right)\right)\right\}_{i \in \mathbb{N}}$.

ОБОЗНАчЕНиЕ 2.11. Так как в силу теоремы 1.37 дифференциалы полиномиальных субримановых дифференциалов во внутреннем базисе имеют вид (5), то при перегруппировке полей по степеням они имеют блочно-диагональный вид. В этом случае каждый блок состоит из единичной матрицы размера $\operatorname{dim} V_{i}$ и либо $\left(\operatorname{dim} \widetilde{V}_{i} \times \operatorname{dim} V_{i}\right)$-блока $(\widehat{D} \varphi)_{\widetilde{V}_{i}, V_{i}}$ матрицы $\widehat{D} \varphi$, либо $\left(\operatorname{dim} \widetilde{V}_{i} \times \operatorname{dim} V_{i}\right)$ блока $D\left(\widehat{D}_{P} \varphi\right)_{\widetilde{V}_{i}, V_{i}}$ матрицы $D\left(\widehat{D}_{P} \varphi\right)$, тогда, перегруппируя при необходимости поля в образе, представим эти блоки как объединение частей $(\widehat{D} \varphi)_{\widetilde{V}_{i}, V_{i}}^{+}$и $(\widehat{D} \varphi)_{\widetilde{V}_{i}, V_{i}}^{-}\left(\right.$соответственно $D\left(\widehat{D}_{P} \varphi\right)_{\widetilde{V}_{i}, V_{i}}^{+}$и $\left.D\left(\widehat{D}_{P} \varphi\right)_{\widetilde{V}_{i}, V_{i}}^{-}\right)$, где «плюсовые» части состоят из строк, соответствующих полям из $\widehat{V}_{i}^{+}$, а «минусовые»- из строк, 
соответствующих полям из $\widehat{V}_{i}^{-}, i=1, \ldots, \widetilde{M}$. Таким образом, для контактных отображений (5) имеет вид

$$
\left(\begin{array}{cccccc}
E_{\operatorname{dim} V_{1}} & 0 & 0 & \ldots & 0 & 0 \\
(\widehat{D} \varphi)_{\widetilde{V}_{1}, V_{1}}^{+}(x) & 0 & 0 & \ldots & 0 & 0 \\
(\widehat{D} \varphi)_{\widetilde{V}_{1}, V_{1}}^{\sigma_{0}}(x) & 0 & 0 & \ldots & 0 & 0 \\
0 & E_{\operatorname{dim} V_{2}} & 0 & \ldots & 0 & 0 \\
0 & (\widehat{D} \varphi)_{\widetilde{V}_{2}, V_{2}}^{+}(x) & 0 & \ldots & 0 & 0 \\
0 & (\widehat{D} \varphi)_{\widetilde{V}_{2}, V_{2}}^{-}(x) & 0 & \ldots & 0 & 0 \\
0 & 0 & E_{\operatorname{dim} V_{3}} & \ldots & 0 & 0 \\
0 & 0 & (\widehat{D} \varphi)_{\widetilde{V}_{3}, V_{3}}^{+}(x) & \ldots & 0 & 0 \\
0 & 0 & (\widehat{D} \varphi)_{\widetilde{V}_{3}, V_{3}}^{-}(x) & \ldots & 0 & 0 \\
\ldots & 0 & 0 & \ldots & 0 & 0 \\
0 & 0 & 0 & \ldots & 0 & (\widehat{D} \varphi)_{\widetilde{V}_{M}, V_{M}}^{+}(x) \\
0 & 0 & 0 & \ldots & 0 & (\widehat{D} \varphi)_{\widetilde{V}_{M}, V_{M}}(x)
\end{array}\right)
$$

с учетом комментариев насчет соотношения $\widetilde{M}$ и $M$ в обозначении 1.36. Для гёльдеровых отображений вид матрицы аналогичный с упомянутыми выше изменениями.

Предположение 2.12. Для $(\widehat{D} \varphi)_{\widetilde{V}_{k}, V_{k}}$ будем полагать, что квадраты длин их столбцов (т. е. евклидовых норм) не превосходят $\frac{1}{\operatorname{dim} V_{k}}-c$ соответственно, $c>0, k=1, \ldots, \widetilde{M}$. Кроме того, будем считать, что $M \geq \widetilde{M}$ и все $\operatorname{dim} V_{1}, \ldots, \operatorname{dim} V_{M}$ положительны.

Свойство 2.13. Из предположения 2.12 следует [30,39], что при $M \geq \widetilde{M}$ и положительных $\operatorname{dim} V_{1}, \ldots, \operatorname{dim} V_{M}$

1) $\operatorname{det}\left(E_{\operatorname{dim} V_{k}}-(\widehat{D} \varphi)_{\widetilde{V}_{k}, V_{k}}^{-}(x)^{*}(\widehat{D} \varphi)_{\widetilde{V}_{k}, V_{k}}^{-}(x)\right) \geq s>0, k=1, \ldots, \widehat{M} ;$

2) квадратичная форма, определяемая матрицей

$$
E_{\operatorname{dim} V_{k}}-(\widehat{D} \varphi)_{\widetilde{V}_{k}, V_{k}}(x)^{*}(\widehat{D} \varphi)_{\widetilde{V}_{k}, V_{k}}(x),
$$

положительно определена, $k=1, \ldots, \widehat{M}$.

ЗАмЕчАниЕ 2.14. При решении задач о метрических характеристиках $\varphi$ утверждения свойства 2.13 можно взять в качестве описания требуемых свойств $\varphi$ (вместо условий предположения 2.12) в зависимости от того, какое утверждение легче проверить.

Свойство 2.15. В условиях предположений 1.18 и 2.12 функция множества $\Phi: A \mapsto S L \mathscr{H}_{\Gamma}^{\nu}\left(\varphi_{\Gamma}(A)\right)$ обладает следующими свойствами:

1) она абсолютно непрерывна относительно меры $\mathscr{H}^{N}$ на $\Omega \subset \mathbb{G}$.

2) она аддитивна на отдаленных шарах.

Теорема 2.16. Пусть выполнены условия предположений 1.18 и 2.12 (или свойства 2.13) и отображение $\varphi: \Omega \rightarrow \widetilde{\mathbb{G}}$ контактно. Тогда поверхность $\varphi_{\Gamma}(\Omega)$ пространственноподобна в смысле определения 2.9 и сублоренцева $S L \mathscr{H}_{\Gamma}^{\nu}$-мера 
образа $\varphi_{\Gamma}(\Omega) \subset \mathbb{U}$ вычисляется по формуле

$$
\int_{\Omega} S L \mathscr{J}(\varphi, v) d \mathscr{H}^{\nu}(v)=\int_{\varphi_{\Gamma}(\Omega)} d^{S L} \mathscr{H}_{\Gamma}^{\nu}(y)
$$

где сублоренцев якобиан $S L \mathscr{J}(\varphi, v)$ равен

$$
\prod_{j=1}^{M} \sqrt{\operatorname{det}\left(E_{\operatorname{dim} V_{j}}+\left(\widehat{D} \varphi^{+}\right)_{\widetilde{V}_{j}, V_{j}}^{*}(v)\left(\widehat{D} \varphi^{+}\right)_{\widetilde{V}_{j}, V_{j}}(v)-\left(\widehat{D} \varphi^{-}\right)_{\widetilde{V}_{j}, V_{j}}^{*}(v)\left(\widehat{D} \varphi^{-}\right)_{\widetilde{V}_{j}, V_{j}}(v)\right)} .
$$

ЗАмечАниЕ 2.17. Определение пространственноподобия, как и в [23], зависит от вторых производных (см. подробности и комментарии в [30] и др.).

ДоКАЗАТЕЛЬСТво ТЕОРЕМЫ 2.16. Общая идея доказательства основана на идеях из [40] (см. также [30]). Опишем основные шаги и прокомментируем идеи доказательства каждого из ключевых утверждений.

ШАГ 1: поверхность $\varphi_{\Gamma}(\Omega)$ пространственноподобна в смысле определения 2.9. Для каждой точки поверхности $\varphi_{\Gamma}(\Omega)$ это утверждение следует из предположения 2.12, что проверяется непосредственными вычислениями (см. [30]). Размер окрестности из определения 2.9 зависит от точности аппроксимации отображения-графика $\varphi_{\Gamma}$ его полиномиальным субримановым дифференциалом, а именно от величины $o(1)$ из определения полиномиальной субримановой дифференцируемости (см. теоремы $1.37,1.38)$.

ШАГ 2: справедливо утверждение свойства 2.15 для функции множества $\Phi$ : $A \mapsto{ }^{S L} \mathscr{H}_{\Gamma}^{\nu}\left(\varphi_{\Gamma}(A)\right), A \subset \Omega$. Это следует из липшицевости отображения-графика и его полиномиального субриманова дифференциала (относительно ${ }^{x} \mathfrak{d}_{2}^{2}$ и адаптированного базиса, см. (5)) и стандартных аргументов, полностью аналогичных приведенным в [30] и др.

ШАГ 3: прообразы сублоренцевых шаров, построенных в адаптированном базисе, при отображении-графике адекватно аппроксимируются прообра-

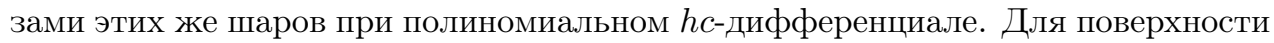
$\varphi_{\Gamma}(\mathscr{U}), \mathscr{U} \Subset \Omega$, выведена следующая характеристика ее аппроксимируемости: при достаточно малых $r>0$ справедливо

$$
\mathscr{H}^{N}\left(\varphi_{\Gamma}^{-1}\left({ }^{v} \operatorname{Box}_{2}^{\mathfrak{d}}\left(\varphi_{\Gamma}(v), r\right)\right)\right)=(1+o(1)) \mathscr{H}^{N}\left(\widehat{D}_{P} \varphi_{\Gamma}(v)^{-1}\left\langle{ }^{v} \operatorname{Box}_{2}^{\mathfrak{d}}\left(\varphi_{\Gamma}(v), r\right)\right\rangle\right),
$$

где $o(1) \rightarrow 0$ при $r \rightarrow 0$ равномерно по $v \in \mathscr{U} \Subset \Omega$. Далее это свойство вместе с явной формулой вычисления меры (см. следующий шаг) применено для вывода локального значения адаптированной меры Хаусдорфа поверхности-графика.

Утверждение этого шага, как и последующих, вытекает из аргументов [30] и др., приведенных почти дословно с очевидными изменениями.

ШАГ 4: мера пересечения образа $\widehat{D}_{P} \varphi_{\Gamma}(v) \mathrm{c}^{v} \mathfrak{d}_{2}^{2}$-шаром явно зависит от значений субриманова дифференциала. Найдено аналитическое выражение $\mathscr{H}^{N}$ меры множества $\widehat{D}_{P} \varphi_{\Gamma}(v)\langle\mathscr{U}\rangle \cap{ }^{v} \operatorname{Box}_{2}^{\mathfrak{d}}\left(\varphi_{\Gamma}(v), r\right)$ с точностью до множителя $1+$ $o(1)$, где $o(1) \rightarrow 0$ при $r \rightarrow 0$ равномерно по $v \in \mathscr{U} \Subset \Omega$ :

$$
\begin{aligned}
\mathscr{H}^{N}\left(\widehat{D}_{P} \varphi_{\Gamma}(v)\langle\mathscr{U}\rangle\right. & \left.\cap{ }^{v} \operatorname{Box}_{2}^{\mathrm{d}}\left(\varphi_{\Gamma}(v), r\right)\right) \\
& =\prod_{j=1}^{M} \omega_{\operatorname{dim} V_{j}} \cdot r^{\nu} \cdot Q(v) \cdot\left|{ }^{v} g\right|_{\widehat{D}_{P} \varphi_{\Gamma}(v)\langle\mathscr{U}\rangle}\left(\varphi_{\Gamma}(v)\right) \mid \cdot(1+o(1)),
\end{aligned}
$$


где ${ }^{v} g$ - риманов тензор в базисе $\left\{{ }^{v} Y_{k}\right\}_{k=1}^{\widehat{N}}$, и

$$
Q(v)=\prod_{j=1}^{M} \frac{\sqrt{\operatorname{det}\left(E_{\operatorname{dim} V_{j}}+(\widehat{D} \varphi)_{\widetilde{V}_{j}, V_{j}}(v)^{*}(\widehat{D} \varphi)_{\widetilde{V}_{j}, V_{j}}(v)\right)}}{\sqrt{\operatorname{det}\left(E_{\operatorname{dim} V_{j}}+(\widehat{D} \varphi)_{\widetilde{V}_{j}, V_{j}}^{+}(v)^{*}(\widehat{D} \varphi)_{\widetilde{V}_{j}, V_{j}}^{+}(v)-\left(\widehat{D}_{\varphi}\right)_{\widetilde{V}_{j}, V_{j}}^{-}(v)^{*}\left(\widehat{D}_{\varphi}\right)_{\widetilde{V}_{j}, V_{j}}^{-}(v)\right)}} .
$$

ШАГ 5: существует прямая связь точной нижней грани сумм из определения 2.10 адаптированной сублоренцевой $S L \mathscr{H}_{\Gamma}^{\nu}$-меры Хаусдорфа для $\varphi_{\Gamma}\left(\operatorname{Box}_{2}(v, r)\right)$ с точной нижней гранью сумм мер покрытия $\operatorname{Box}_{2}(v, r)$ прообразами сублоренцевых шаров (из этого же определения) при отображении $\varphi_{\Gamma}$. Установлено, что точная нижняя грань сумм вида $\sum_{i \in \mathbb{N}} r_{i}^{\nu}$ из определения 2.10 достигается тогда и только тогда, когда значение $\sum_{j \in \mathbb{N}} \mathscr{H}^{N}\left(\varphi_{\Gamma}^{-1}\left(v_{j} \operatorname{Box}_{2}^{\mathfrak{d}}\left(\varphi_{\Gamma}\left(v_{j}\right), r_{j}\right)\right)\right)$ близко с точностью до множителя $1+o(1)$ к точной нижней грани, равной $\mathscr{H}^{N}\left(\operatorname{Box}_{2}(v, r)\right)$, где шары $\left\{v_{j} \operatorname{Box}_{2}^{\mathfrak{d}}\left(\varphi_{\Gamma}\left(v_{j}\right), r_{j}\right)\right\}_{j \in \mathbb{N}}, r_{j}<\delta, j \in \mathbb{N}$, покрывают множество $\varphi_{\Gamma}\left(\operatorname{Box}_{2}(v, r)\right)$ и $o(1) \rightarrow 0$ при $\delta \rightarrow 0$ равномерно по $v \in \mathscr{U} \Subset \Omega(\mathrm{cm}$. также [30]).

ШАГ 6: выведена производная функции множества $\Phi^{\prime}(v)=S L \mathscr{J}(\varphi, v)$, $v \in \Omega$, с помощью которой доказана формула площади (6).

В силу теоремы 1.38 справедлив следующий результат для графиков достаточно гладких неконтактных отображений.

Теорема 2.18. Пусть выполнены условия предположений 1.18, 2.12 (или свойства 2.13) с учетом замены элементов $\widehat{D} \varphi(x)\langle y\rangle$ соответствующими элементами $\widehat{D}_{P} \varphi(x)\langle y\rangle$ в условиях теоремы 1.38. Тогда поверхность $\varphi_{\Gamma}(\Omega)$ пространственноподобна в смысле определения 2.9 и сублоренцева ${ }^{S L} \mathscr{H}_{\Gamma}^{\nu}$-мера образа $\varphi_{\Gamma}(\Omega) \subset \mathbb{U}$ вычисляется по формуле

$$
\int_{\Omega} S L \mathscr{J}(\varphi, v) d \mathscr{H}^{\nu}(v)=\int_{\varphi_{\Gamma}(\Omega)} d^{S L} \mathscr{H}_{\Gamma}^{\nu}(y),
$$

где сублоренцев якобиан $S L \mathscr{J}(\varphi, v)$ равен

$$
\begin{aligned}
\prod_{j=1}^{M}\left(\operatorname { d e t } \left(E_{\operatorname{dim} V_{j}}+D\left(\widehat{D}_{P} \varphi^{+}\right)_{\widetilde{V}_{j}, V_{j}}^{*}(v)\right.\right. & D\left(\widehat{D}_{P} \varphi^{+}\right)_{\widetilde{V}_{j}, V_{j}}(v) \\
& \left.\left.-D\left(\widehat{D}_{P} \varphi^{-}\right)_{\widetilde{V}_{j}, V_{j}}^{*}(v) D\left(\widehat{D}_{P} \varphi^{-}\right)_{\widetilde{V}_{j}, V_{j}}(v)\right)\right)^{1 / 2}
\end{aligned}
$$

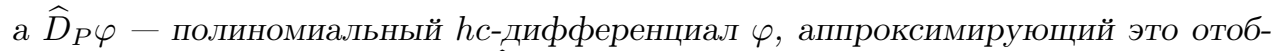
ражение с точностью до о $\left(d_{2}\right)^{\widehat{M}}$.

ДокАЗАтЕльство. Все аргументы следуют той же схеме, что и вывод (6), с учетом теоремы 1.38 и аналога свойства 2.13 для $D\left(\widehat{D}_{P} \varphi\right)_{\widetilde{V}_{j}, V_{j}}^{-}, j=1, \ldots$, $\min \{M, \widetilde{M}\}$.

Рассмотрим класс $C_{H}^{1}$-отображений групп Карно в нильпотентные градуированные группы, представляющий независимый интерес. Подчеркнем, что в этом случае $\varphi$ может не быть дифференцируемым в классическом смысле. 
Предположение 2.19. Для $y \in \Omega$ определим

$$
\varphi_{y}(x)=\exp \left(\sum_{j=1}^{\widetilde{N}} \varphi_{j}(x) \widetilde{X}_{j}\right)(y)
$$

и рассмотрим точку

$$
\exp \left(\sum_{j=1}^{\widetilde{N}} \zeta_{j} \widetilde{X}_{j}\right)\left(\varphi_{y}(x)\right)
$$

где $\zeta=\left(\zeta_{1}, \ldots, \zeta_{\widetilde{N}}\right)$ принадлежит некоторой окрестности нуля в $\mathbb{R}^{\widetilde{N}}$. Далее будем считать, что в каждой точке $x$ выполняется одно из следующих условий:

1) существует замена исходного базиса адаптированным, приводящая координаты точки (7) относительно $\varphi_{\Gamma}(x)$ к виду

$$
\left\{\begin{array}{l}
\Delta_{i}(x, y)=y_{i}+\sum_{\substack{\alpha+\tau||_{h}=\operatorname{deg} X_{i}, \alpha, \tau>0}} \widehat{L}_{\alpha, \tau}^{X_{i}}(x) y^{\alpha} \zeta^{\tau}, \quad i=1, \ldots, N \\
\widetilde{\Delta}_{j}(x, y)=\zeta_{j}+\sum_{\substack{|\alpha+\tau|_{h}=\operatorname{deg} \widetilde{X}_{j}, \alpha, \tau>0}} \widehat{L}_{\alpha, \tau} \widetilde{X}_{j}(x) y^{\alpha} \zeta^{\tau}, \quad j=1, \ldots, \widetilde{N}
\end{array}\right.
$$

2) существует замена исходного базиса адаптированным, приводящая (4) к виду

$$
\begin{cases}\Delta_{i}(x, y)=y_{i}+\sum_{\substack{|\alpha+\tau|_{h}=\operatorname{deg} X_{i}, \alpha, \tau>0}} \widehat{L}_{\alpha, \tau}^{X_{i}}(x) y^{\alpha}(\widehat{D} \varphi(x)\langle y\rangle)^{\tau}, & i=1, \ldots, N, \\ \widetilde{\Delta}_{j}(x, y)=(\widehat{D} \varphi(x)\langle y\rangle)_{j}+\sum_{\substack{|\alpha+\tau|_{h}=\operatorname{deg} \widetilde{X}_{j}, \alpha, \tau>0}} \widehat{L}_{\alpha, \tau}^{\widetilde{X}_{j}}(x) y^{\alpha}(\widehat{D} \varphi(x)\langle y\rangle)^{\tau}, & j=1, \ldots, \widetilde{N},\end{cases}
$$

причем внутреннее расстояние ${ }^{x} \hat{d}_{2}$ на $\mathbb{U}$, ограниченное на проходящее через $y \in$ $\Omega$ интегральное многообразие $\widetilde{\mathbb{G}}_{y}$ распределения $\left\{\widetilde{X}_{j}\right\}_{j=1}^{\widetilde{N}}$, локально билипшицево эквивалентно $\tilde{d}_{2}$.

Будем считать, что помимо выполнения одного из этих условий такая замена сохраняет на $\mathbb{U}$ треугольную таблицу коммутаторов с исходными степенями базисных полей: справедливы соотношения

$$
\left[{ }^{x} Y_{i},{ }^{x} Y_{j}\right](y)=\sum_{k: \operatorname{deg} Y_{k} \leq \operatorname{deg} Y_{i}+\operatorname{deg} Y_{j}} \hat{c}_{i j k}(x) \cdot{ }^{x} Y_{k}(y)
$$

для всех $i, j=1, \ldots, \widehat{N}$. Кроме того, будем считать, что $M \geq \widetilde{M}$.

ЗАМЕЧАНИЕ 2.20. Если в (4)

1) $K_{\kappa, \mu, \lambda}^{Y} \neq 0$ только при $|\kappa|=1$,

2) $L_{\alpha, \beta, \gamma, \tau}^{\widetilde{X}_{j}} \neq 0$ только при $\beta=\gamma=0$, 
то координаты (7) будут иметь вид

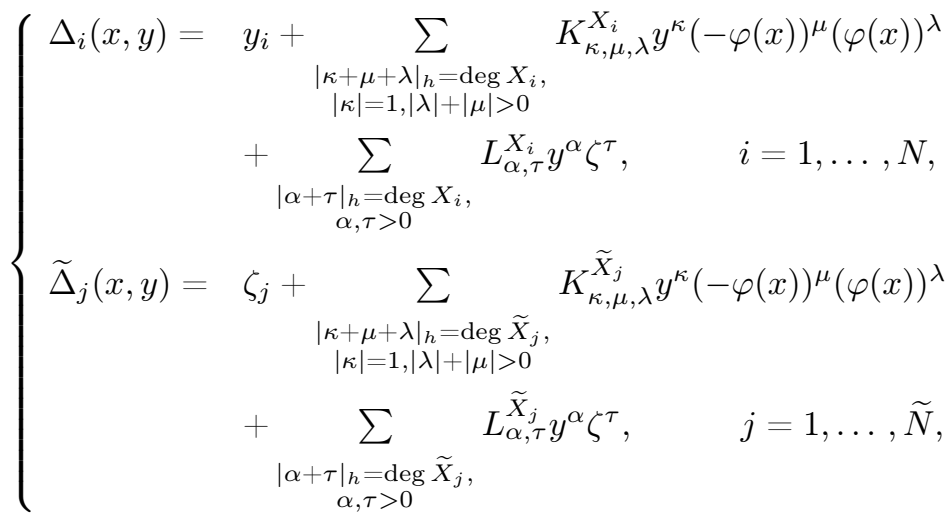

$y=\left(y_{1}, \ldots, y_{N}\right), \varphi(x)=\left(\varphi_{1}(x), \ldots, \varphi_{\widetilde{N}}(x)\right)$, a $K_{\kappa, \mu, \lambda}^{X_{i}}, L_{\alpha, \tau}^{X_{i}}, K_{\kappa, \mu, \lambda}^{\widetilde{X}_{j}}$ и $L_{\alpha, \tau}^{\widetilde{X}_{j}}$ - константы для всех мультииндексов $\kappa, \mu, \lambda, \alpha, \tau$. Тогда для получения соотношений (8) (в частности, для приведения (4) к виду (9)) достаточно применить линейные преобразования базиса. Заметим, что в этом случае меняются только поля $X_{1}, \ldots, X_{n_{M-1}}$.

ПримеР 2.21. В [30] рассмотрен случай $C_{H}^{1}$-отображений двуступенчатой группы Карно в двуступенчатую нильпотентную градуированную группу. Такие отображения удовлетворяют условиям предположения 2.19.

Другими примерами отображений, для которых выполняется условие 2.19, являются следующие случаи.

(1) $\left[X_{i}, \widetilde{X}_{j}\right]=0$ на $\mathbb{U}, i=1, \ldots, N, j=1, \ldots, \widetilde{N}$; в этом случае формула $(4)$ имеет вид

$$
\begin{cases}\Delta_{i}(x, y)=y_{i}, & i=1, \ldots, N \\ \widetilde{\Delta}_{j}(x, y)=(\widehat{D} \varphi(x)\langle y\rangle)_{j}, & j=1, \ldots, \widetilde{N} .\end{cases}
$$

(2) Пусть $\left[\widetilde{X}_{j}, X_{i}\right] \in \widehat{V}_{\widehat{M}}, i=1, \ldots, N, j=1, \ldots, \widetilde{N}$. Тогда координаты $(7)$ относительно $\varphi_{\Gamma}(x)$ равны

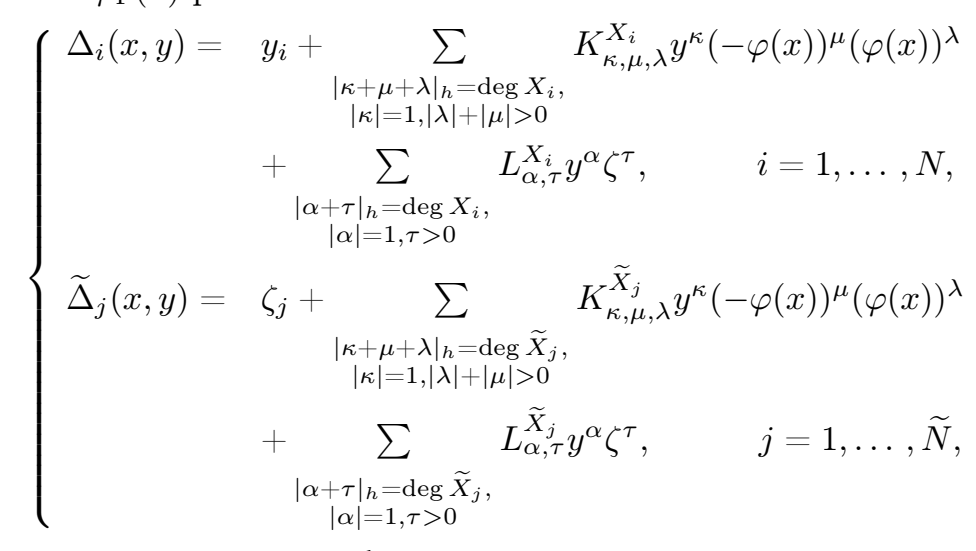

и, кроме того, треугольная таблица коммутаторов после приведения коэффициентов полиномиального субриманова дифференциала к виду (9) сохраняется, так как коммутаторы добавленных к исходным полей будут нулевыми по выбору условия. Утверждение проверяется непосредственно формулой Бейкера Кэмпбелла - Хаусдорфа. Поля, входящие в алгебру Ли группы $\widetilde{G}$, остаются без изменений. 
Свойство 2.22. Отображения, удовлетворяющие условиям предположения 2.19, аппроксимируются полиномиальным субримановым дифференциалом (9) относительно ${ }^{x} \hat{d}_{2}$. Кроме того, координата $\varphi_{\Gamma}(y)$ относительно $\varphi_{\Gamma}(x)$, соответствующая полю ${ }^{x} Y_{k}$, отличается от аналогичной координаты $\widehat{D}_{P} \varphi_{\Gamma}(x)\langle y\rangle$ на величину $о\left(d_{2}(x, y)^{\operatorname{deg} Y_{k}}\right)$, где $Y_{k}$ - базисное поле из набора $\left\{X_{i}\right\}_{i=1}^{N}$ или $\left\{\widetilde{X}_{j}\right\}_{j=1}^{\widetilde{N}}, k=1, \ldots, \widehat{N}$.

ДокАЗАТЕЛЬСТво. Если выполнено условие 2 предположения 2.19, то утверждение об аппроксимируемости следует из предположения о локальной билипшицевой эквивалентности расстояний. Утверждение о координатах следует из сохранения треугольной таблицы коммутаторов. Действительно, в этом случае групповая операция в адаптированном базисе имеет тот же вид, что и в исходном, с точностью до структурных констант.

Пусть теперь выполнено условие 1 предположения 2.19. Обозначим через $\left(\varphi_{1}^{\Delta}\left(x_{\rightarrow} y\right), \ldots, \varphi \underset{\widetilde{N}}{\Delta}\left(x_{\rightarrow} y\right)\right)$ такой набор координат, что

$$
\varphi(y)=\exp \left(\sum_{j=1}^{\tilde{N}} \varphi_{j}^{\Delta}\left(x_{\rightarrow} y\right) \widetilde{X}_{j}\right)(\varphi(x)) .
$$

По свойству $h c$-дифференцируемости имеем

$$
\left|\varphi_{j}^{\Delta}\left(x_{\rightarrow} y\right)-(\widehat{D} \varphi(x)\langle y\rangle)_{j}\right|=o\left(d_{2}(x, y)^{\operatorname{deg} \widetilde{X}_{j}}\right),
$$

$j=1, \ldots, \tilde{N}$. Кроме того, по предположению $\varphi_{\Gamma}(y)$ в новом базисе имеет координаты

$$
\begin{cases}\Phi_{i}(x, y)=y_{i}+\sum_{\substack{|\alpha+\tau|_{h}=\operatorname{deg} X_{i}, \alpha, \tau>0}} \widehat{L}_{\alpha, \tau}^{X_{i}}(x) y^{\alpha}\left(\varphi^{\Delta}\left(x_{\rightarrow} y\right)\right)^{\tau}, & i=1, \ldots, N, \\ \widetilde{\Phi}_{j}(x, y)=\varphi_{j}^{\Delta}\left(x_{\rightarrow} y\right)+\sum_{\substack{|\alpha+\tau|_{h}=\operatorname{deg} \widetilde{X}_{j}, \tau>0 \\ \alpha, \tau>0}} \widehat{L}_{\alpha, \tau}^{\widetilde{X}_{j}}(x) y^{\alpha}\left(\varphi^{\Delta}\left(x_{\rightarrow} y\right)\right)^{\tau}, \quad j=1, \ldots, \widetilde{N},\end{cases}
$$

где $\varphi^{\Delta}\left(x_{\rightarrow} y\right)=\left(\varphi_{1}^{\Delta}\left(x_{\rightarrow} y\right), \ldots, \varphi_{\widetilde{N}}^{\Delta}\left(x_{\rightarrow} y\right)\right)$. Для доказательства точности аппроксимации оценим разности $\left|\Delta_{i}(x, y)-\Phi_{i}(x, y)\right|$ и $\left|\widetilde{\Delta}_{j}(x, y)-\widetilde{\Phi}_{j}(x, y)\right|$ и покажем, что они равны $o\left(d_{2}(x, y)^{\operatorname{deg} X_{i}}\right)$ и $o\left(d_{2}(x, y)^{\operatorname{deg} \widetilde{X}_{j}}\right)$ соответственно, $i=$ $1, \ldots, N, j=1, \ldots, \widetilde{N}$. Для $\operatorname{deg} X_{i}=1$ утверждение следует из (11). Для $\operatorname{deg} X_{i}=2$ выводим

$$
\begin{array}{r}
\left|\Delta_{i}(x, y)-\Phi_{i}(x, y)\right| \leq \sum_{\substack{|\alpha+\tau|_{h}=2, \alpha, \tau>0}}\left|\widehat{L}_{\alpha, \tau}^{X_{i}}(x)\right| \cdot\left|y^{\alpha}\right| \cdot\left|(\widehat{D} \varphi(x)\langle y\rangle)^{\tau}-\left(\varphi^{\Delta}\left(x_{\rightarrow} y\right)\right)^{\tau}\right| \\
=O\left(d_{2}(x, y)\right) \cdot o\left(d_{2}(x, y)\right)=o\left(d_{2}(x, y)^{2}\right) .
\end{array}
$$

Если утверждение верно для $|\alpha+\tau|_{h}=k, k \geq 2$, то случай $|\alpha+\tau|_{h}=k+1$ сводится к доказанному ранее. Действительно, если $|\tau|=1$, то $\operatorname{deg} X_{\tau}=k+$ $1-|\alpha|_{h}$, поэтому утверждение является следствием (11). Пусть теперь $|\tau|>1$. В этом случае $\tau=\tau_{1}+\tau_{2}$, где $\tau_{1}, \tau_{2} \neq 0$, и так как $\left|\alpha+\tau_{1}\right|_{h} \leq k$ и $\left|\alpha+\tau_{2}\right|_{h} \leq k$, то

$$
\begin{aligned}
\mid(\widehat{D} \varphi(x)\langle y\rangle)^{\tau_{1} \tau_{2}} & -\left(\varphi^{\Delta}\left(x_{\rightarrow} y\right)\right)^{\tau_{1} \tau_{2}} \mid \\
& \leq\left|(\widehat{D} \varphi(x)\langle y\rangle)^{\tau_{1}}\right| \cdot\left|(\widehat{D} \varphi(x)\langle y\rangle)^{\tau_{2}}-\left(\varphi^{\Delta}\left(x_{\rightarrow} y\right)\right)^{\tau_{2}}\right| \\
+ & \left|\left(\varphi^{\Delta}\left(x_{\rightarrow} y\right)\right)^{\tau_{2}}\right| \cdot\left|(\widehat{D} \varphi(x)\langle y\rangle)^{\tau_{1}}-\left(\varphi^{\Delta}\left(x_{\rightarrow} y\right)\right)^{\tau_{1}}\right|=o\left(d_{2}(x, y)^{|\tau|_{h}}\right) .
\end{aligned}
$$


Следовательно, $\left|\Delta_{i}(x, y)-\Phi_{i}(x, y)\right|=o\left(d_{2}(x, y)^{\operatorname{deg} X_{i}}\right)$. Случаи для $j=1, \ldots, \tilde{N}$ доказываются аналогичными аргументами.

Так как по условию адаптированный базис сохраняет треугольную таблицу коммутаторов с исходными степенями (см. (10)), формулы групповой операции для новых полей имеют тот же вид, что и для исходных; разница состоит только в структурных константах. Поэтому дальнейшие рассуждения в доказательстве того, что

$$
{ }^{x} \hat{d}_{2}\left(\varphi_{\Gamma}(y), \widehat{D}_{P} \varphi_{\Gamma}(x)\langle y\rangle\right)=o\left(d_{2}(x, y)\right),
$$

стандартны и основываются на формулах групповой операции (3). Свойство доказано.

ОПРЕДЕЛЕНИЕ 2.23. Степень полинома, подсчитанную относительно растяжений

$$
\left(y_{1}, \ldots, y_{N}\right) \mapsto \delta_{\varepsilon}\left(y_{1}, \ldots, y_{N}\right)=\left(\varepsilon^{\operatorname{deg} X_{1}} y_{1}, \ldots, \varepsilon^{\operatorname{deg} X_{N}} y_{N}\right)
$$

будем называть однородной.

Следующее утверждение доказывает формулу площади для отображенийграфиков, построенных по отображениям класса $C_{H}^{1}$, которые, в общем случае, не являются дифференцируемыми в классическом смысле.

Теорема 2.24. Пусть выполнены условия предположений 1.18, пп. (1)(5), и 2.12 (или свойства 2.13), $M \geq \widetilde{M}$, отображкение $\varphi: \Omega \rightarrow \widetilde{\mathbb{G}}$ принадлежит классу $C_{H}^{1}$ и, кроме того, $\widehat{V}_{k}^{-}=\widetilde{V}_{k}, k=2, \ldots, \widetilde{M}$. Тогда существует такая нормированная мера $S L \mathscr{H}_{\mathfrak{b}}^{\nu}$, вычисляемая явно через значения полиномиального субриманова дифференциала, для которой справедлива формула площади

$$
\int_{\Omega} S L \mathscr{J}(\varphi, x) d \mathscr{H}^{\nu}(x)=\int_{\varphi_{\Gamma}(\Omega)} d^{S L} \mathscr{H}_{\mathfrak{b}}^{\nu}(y),
$$

где сублоренцев якобиан ${ }^{S L} \mathscr{J}(\varphi, x)$ равен

$$
\begin{array}{r}
\sqrt{\operatorname{det}\left(E_{\operatorname{dim} V_{1}}+\left(\widehat{D} \varphi^{+}\right)_{\widetilde{V}_{1}, V_{1}}^{*}(x)\left(\widehat{D} \varphi^{+}\right)_{\widetilde{V}_{1}, V_{1}}(x)-\left(\widehat{D} \varphi^{-}\right)_{\widetilde{V}_{1}, V_{1}}^{*}(x)\left(\widehat{D} \varphi^{-}\right)_{\widetilde{V}_{1}, V_{1}}(x)\right)} \\
\times \prod_{j=2}^{M} \sqrt{\operatorname{det}\left(E_{\operatorname{dim} V_{j}}-\left(\widehat{D} \varphi^{-}\right)_{\widetilde{V}_{j}, V_{j}}^{*}(x)\left(\widehat{D} \varphi^{-}\right)_{\widetilde{V}_{j}, V_{j}}(x)\right)} .
\end{array}
$$

ДокАЗАТЕЛЬСтво данной теоремы принципиально отличается от доказательства теоремы 2.16 на шагах 1 и 4 в силу разницы в структуре полиномиального субриманова дифференциала. Поэтому опишем только эти шаги (остальные проводятся по представленной выше схеме с очевидными изменениями).

ШАГ 1. Пусть $x \in \Omega$. Покажем, что существует такая окрестность $\mathscr{U} \subset \Omega$, $\mathscr{U} \ni x$, что для поверхности $\varphi_{\Gamma}(\mathscr{U})$ будут выполнены условия определения 2.9 . У тверждение тривиально, если $M>\widetilde{M}$ и в этом случае условие определения 2.9 справедливо для $y_{\operatorname{dim}} V_{\widetilde{M}}+1, \ldots, y_{\operatorname{dim}} V_{\widetilde{M}+1}$ или для переменных большей степени, где $y=\exp \left(\sum_{i=1}^{N} y_{i} X_{i}\right)(x), x \in \mathscr{U}$, а $y$ - точка из достаточно малой окрестности $x$, также лежащая в $\mathscr{U}$. В противном случае рассуждения такие же, как и для $M=\widetilde{M}$.

Пусть $M=\widetilde{M}$. 
Обозначим $\left(\delta_{1}(x, y), \ldots, \delta_{\widehat{N}}(x, y)\right)={ }^{x} \tilde{\theta}_{\varphi_{\Gamma}(x)}^{-1}\left(\varphi_{\Gamma}(y)\right)$ (см. теорему 1.37). На достаточно малой окрестности $\mathscr{U}$ для ее точек $x, y$ в силу предположения 2.12 (или п. 2 свойства 2.13) выполнено неравенство

$$
\sum_{j=1}^{\operatorname{dim} \widehat{V}_{1}^{+}} \delta_{j}(x, y)^{2}-\sum_{l=\operatorname{dim} \widehat{V}_{1}^{+}+1}^{\operatorname{dim} \widehat{V}_{1}} \delta_{l}(x, y)^{2} \geq\left(c \operatorname{dim} V_{1}\right) \sum_{j=1}^{\operatorname{dim} V_{1}} y_{j}^{2}-T_{1} \varepsilon\left(d_{2}(x, y)\right)^{2},
$$

где $T_{1}<\infty$ зависит от $\widehat{D} \varphi$, и оно равномерно на $\mathscr{U}$. Если правая часть $(12)$ положительна, то требуемое условие выполняется.

Рассмотрим случай, когда правая часть (12) не превосходит 0. Из (9) следует, что для $y=\exp \left(\sum_{i=1}^{N} y_{i} X_{i}\right)(x)$ действие $\widehat{D}_{P} \varphi_{\Gamma}(x)$ на этом элементе в нормальных координатах ${ }^{x} \tilde{\theta}_{\varphi_{\Gamma}(x)}^{-1}$ представимо как умножение матрицы

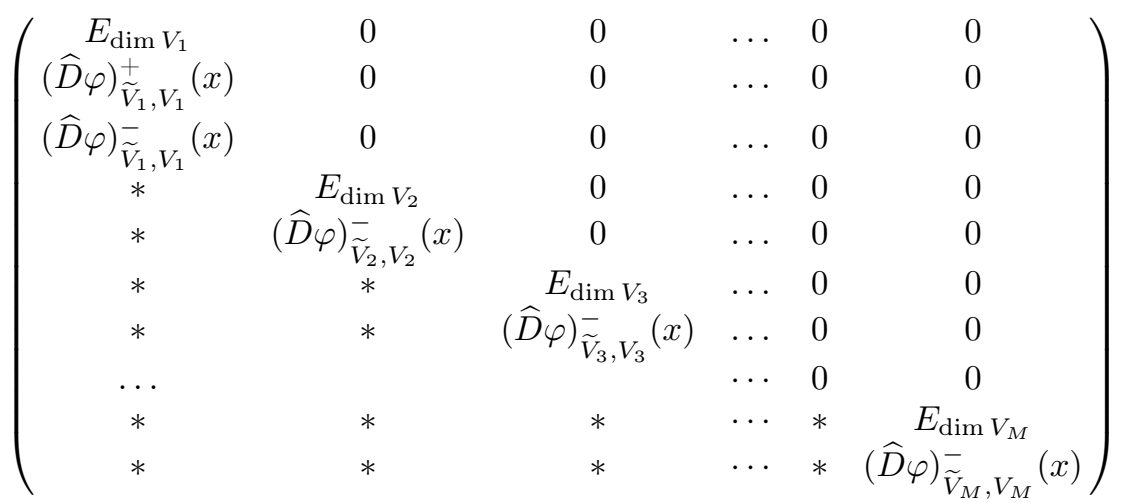

на вектор $\left(y_{1}, \ldots, y_{N}\right)^{T}$. Здесь на месте каждого элемента «*» в строке $m$ и столбце $l$ находится полином от $\left(y_{1}, \ldots, y_{N}\right)$, являющийся однородным относительно растяжений

$$
\left(y_{1}, \ldots, y_{N}\right) \mapsto \delta_{\varepsilon}\left(y_{1}, \ldots, y_{N}\right)=\left(\varepsilon^{\operatorname{deg} X_{1}} y_{1}, \ldots, \varepsilon^{\operatorname{deg} X_{N}} y_{N}\right) .
$$

Иными словами, каждому элементу полинома вида $y^{\mu}$ можно сопоставить значение $|\mu|_{h}$ (см. также определение 1.9), которое не меняется. В этом случае однородная степень полинома, посчитанная таким образом, равна $|\mu|_{h}=$ $\operatorname{deg} Y_{m}-\operatorname{deg} X_{l}$. Подчеркнем, что считаем поля $Y_{1}, \ldots, Y_{\widehat{N}}$ упорядоченными по степеням. Фиксируем $k>1$. Рассмотрим произвольный блок

$$
\left(\begin{array}{c}
E_{\operatorname{dim} V_{k}} \\
(\widehat{D} \varphi)_{\widetilde{V}_{k}, V_{k}}^{\tilde{x}_{k}}(x)
\end{array}\right)
$$

и оценим разность вида (12) для соответствующего блока матрицы (13)

$$
\left(\begin{array}{cccccc}
* & * & E_{\operatorname{dim} V_{k}} & 0 & \ldots & 0 \\
* & * & (\widehat{D} \varphi)_{\widetilde{V}_{k}, V_{k}}^{-}(x) & 0 & \ldots & 0
\end{array}\right),
$$

который действует на $\left(y_{1}, \ldots, y_{n_{k}}\right)$. Заметим, что это действие можно представить как

$$
\left(\begin{array}{c}
E_{\operatorname{dim} V_{k}} \\
(\widehat{D} \varphi)_{\widetilde{V}_{k}, V_{k}}^{\bar{N}_{k}}(x)
\end{array}\right)\left(\begin{array}{c}
y_{n_{k-1}+1} \\
\vdots \\
y_{n_{k}}
\end{array}\right)+\left(\begin{array}{lll}
c_{1} & \ldots & c_{n_{k-1}}
\end{array}\right)\left(\begin{array}{c}
y_{1} \\
\vdots \\
y_{n_{k-1}}
\end{array}\right),
$$


где элементы столбца $c_{j}$ сравнимы с $O\left(y^{\mu_{j}}\right),\left|\mu_{j}\right|_{h}=k-\operatorname{deg} X_{j}$. Для доказательства пространственноподобия необходимо проанализировать соотношение

$$
\begin{aligned}
\sum_{i=n_{k-1}+1}^{n_{k}} y_{i}^{2}- & \left.\sum_{j=1}^{\operatorname{dim} \widetilde{V}_{k}}((\widehat{D} \varphi))_{\widetilde{V}_{k}, V_{k}}(x)\left\langle y_{n_{k-1}+1}, \ldots, y_{n_{k}}\right\rangle\right)_{j}^{2} \\
& -P^{k}\left(y_{1}, \ldots, y_{n_{k}}\right)-T_{k} \varepsilon d_{2}(x, y)^{2 k}
\end{aligned}
$$

где значения $P^{k}$ определяются значениями во втором слагаемом в (14) и их произведениями с первым слагаемым, его однородная степень равна $2 k$, a $T_{k}<\infty$ зависит от $\widehat{D} \varphi$ и констант из (9) равномерно на $\mathscr{U}$. В силу предположения 2.12 имеем

$$
\sum_{i=n_{k-1}+1}^{n_{k}} y_{i}^{2}-\sum_{j=1}^{\operatorname{dim} \widetilde{V}_{k}}\left((\widehat{D} \varphi) \overline{\widetilde{V}}_{k}, V_{k}(x)\left\langle y_{n_{k-1}+1}, \ldots, y_{n_{k}}\right\rangle\right)_{j}^{2} \geq c \operatorname{dim} V_{k} \sum_{i=n_{k-1}+1}^{n_{k}} y_{i}^{2} .
$$

Оценим значение полинома

$$
P^{k}\left(y_{1}, \ldots, y_{n_{k}}\right)=\sum_{\alpha:|\alpha|_{h}=2 k} K_{\alpha}(x) y^{\alpha},
$$

коэффициенты которого локально равномерно ограничены. Напомним, что в этом полиноме степень $y_{n_{k-1}+1}, \ldots, y_{n_{k}}$ не превосходит 1. Обозначим

$$
\mathbf{y}_{l}^{2}=\sum_{i=n_{l-1}+1}^{n_{l}} y_{i}^{2}, \quad l=1, \ldots, M, \quad n_{0}=0, \quad \mathbf{y}=\left(\mathbf{y}_{1}, \ldots, \mathbf{y}_{M}\right) .
$$

Заметим, что в этом случае $d_{2}(x, y)=\max _{m=1, \ldots, M}\left\{\mathbf{y}_{m}^{1 / m}\right\}$. Полином $P^{k}$ в силу неравенства Гёльдера можно оценить величиной

$$
\left|\sum_{\alpha:|\alpha|_{h}=2 k} K_{\alpha}(x) y^{\alpha}\right| \leq C \cdot \mathbf{y}_{k} \sum_{\substack{\left|\beta_{1}+\beta_{2}\right|_{h}=k, \beta_{1}, \beta_{2}>0}} \mathbf{y}^{\beta_{1}+\beta_{2}}+C \cdot \sum_{\substack{\left|\lambda_{1}+\lambda_{2}\right|_{h}=k,\left|\lambda_{3}+\lambda_{4}\right|_{h}=k, \lambda_{1}, \lambda_{2}, \lambda_{3}, \lambda_{4}>0}} \mathbf{y}^{\lambda_{1}+\lambda_{2}+\lambda_{3}+\lambda_{4}} .
$$

Следовательно, (15) оценивается снизу величиной

$$
c \operatorname{dim} V_{k} \mathbf{y}_{k}^{2}-C \cdot \mathbf{y}_{k} \sum_{\beta:|\beta|_{h}=k} \mathbf{y}^{\beta}-C \cdot \sum_{\lambda:|\lambda|_{h}=2 k} \mathbf{y}^{\lambda}-T_{k} \varepsilon \max _{m=1, \ldots, M}\left\{\mathbf{y}_{m}^{2 k / m}\right\},
$$

где $\beta=\beta_{1}+\beta_{2}$ и $\lambda=\lambda_{1}+\lambda_{2}+\lambda_{3}+\lambda_{4}$, и такой вывод справедлив для всех $k=$ $2, \ldots, M$. Для доказательства пространственноподобия достаточно, чтобы (16) была положительна хотя бы для одного $k=2, \ldots, M$.

Предположим, что это не так. Напомним, что по условию рассматриваемого случая имеем

$$
\mathbf{y}_{1}^{2} \leq \frac{T_{1} \varepsilon}{c \operatorname{dim} V_{1}} \max _{m=1, \ldots, M}\left\{\mathbf{y}_{m}^{2 / m}\right\}
$$

Тогда для $k=2$ выводим следующую оценку снизу и предполагаем, что она неположительна:

$$
\mathbf{y}_{2}^{2}-\mathbf{y}_{2} \cdot C_{1,2} \varepsilon \max _{m=1, \ldots, M}\left\{\mathbf{y}_{m}^{2 / m}\right\}-C_{2,2} \varepsilon \max _{m=1, \ldots, M}\left\{\mathbf{y}_{m}^{4 / m}\right\} \leq 0,
$$

поэтому $\mathbf{y}_{2}<C_{3,2} \varepsilon^{1 / 2} \max _{m=1, \ldots, M}\left\{\mathbf{y}_{m}^{2 / m}\right\}$. 
Применяя аналогичные рассуждения для $k=3, \ldots, M$, приходим к выводу, что

$$
\mathbf{y}_{k}<C_{3, k} \varepsilon^{\prime} \max _{m=1, \ldots, M}\left\{\mathbf{y}_{m}^{k / m}\right\}, \quad k=1, \ldots, M,
$$

где величина $\varepsilon^{\prime}$ достаточно мала и зависит от аппроксимации отображения субримановым дифференциалом, а $C_{3, k}$ локально равномерно ограничена и не зависит от $\varepsilon^{\prime}$. Иными словами, получено противоречие:

$$
\max _{m=1, \ldots, M}\left\{\mathbf{y}_{m}^{1 / m}\right\}<C_{0} \tilde{\varepsilon} \max _{m=1, \ldots, M}\left\{\mathbf{y}_{m}^{1 / m}\right\},
$$

где произведение $C_{0} \widetilde{\varepsilon}$ может быть сколь угодно малым. Таким образом, хотя бы для одного $k \in[1, M]$ величина (16) и, следовательно, (15) положительна, поэтому поверхность $\varphi_{\Gamma}(\Omega)$ пространственноподобна.

ШАГ 4. Пусть $\mathscr{H}_{x}^{l}-l$-мерная мера Хаусдорфа, построенная относительно адаптированного в $x$ базиса, $l \in \mathbb{N}$. Вычислим в координатах первого рода относительно $\varphi_{\Gamma}(x)$ (построенных относительно адаптированного в точке $x$ базиса) $\mathscr{H}_{x}^{N}$-меру пересечения $B$ образа полиномиального субриманова дифференциала $\widehat{D}_{P} \varphi_{\Gamma}(x)\langle\mathscr{U}\rangle$ и сублоренцева шара ${ }^{x} \operatorname{Box}_{2}^{\mathfrak{o}}\left(\varphi_{\Gamma}(x), r\right), x \in \mathscr{U} \Subset \Omega$. Чтобы не усложнять обозначения, действующее в координатах первого рода отображение обозначим также символом $\varphi$, а соответствующий график - символом $\varphi_{\Gamma}$. В прообразе при необходимости будем тоже рассматривать координаты первого рода относительно $x$.

Структура (13) позволяет последовательно применить формулу коплощади для отображения проекции $\pi_{1}$ сначала на горизонтальное подпространство $\widehat{V}_{1}$, затем соответственно $\pi_{2}, \ldots, \pi_{\widehat{M}-1}$ последовательно на $\widehat{V}_{2}, \ldots, \widehat{V}_{\widehat{M}-1}$ (структура в координатах первого рода позволяет отождествлять точки множества $\widehat{V}_{k}$ и $\left.\exp \left(\widehat{V}_{k}\right)(0), k=1, \ldots, \widehat{M}\right)$ :

$$
\begin{gathered}
\int_{B} d \mathscr{H}_{x}^{N}=\int_{B} \mathscr{J}_{\operatorname{dim} \widehat{V}_{1}}\left(\pi_{1}\right) d \mathscr{H}_{x}^{N}=\int_{\widehat{V}_{1}} d \mathscr{H}_{x}^{\operatorname{dim} V_{1}}\left(y_{\widehat{V}_{1}}\right) \int_{\pi_{1}^{-1}\left(y_{\widehat{V}_{1}}\right) \cap B} d \mathscr{H}_{x}^{N-\operatorname{dim} V_{1}}(y) \\
=\int_{\widehat{V}_{1}} d \mathscr{H}_{x}^{\operatorname{dim} V_{1}}\left(y_{\widehat{V}_{1}}\right) \int_{\widehat{V}_{2}} d \mathscr{H}_{x}^{\operatorname{dim} V_{2}}\left(y_{\widehat{V}_{2}}\right) \int_{\pi_{2}^{-1}\left(y_{\widehat{V}_{2}}\right) \cap B} d \mathscr{H}_{x}^{N-\operatorname{dim} V_{1}-\operatorname{dim} V_{2}}(y) \\
=\int_{\widehat{V}_{1}} d \mathscr{H}_{x}^{\operatorname{dim} V_{1}}\left(y_{\widehat{V}_{1}}\right) \cdots \int_{\widehat{V}_{M-1}} d \mathscr{H}_{x}^{\operatorname{dim} V_{M-1}\left(y_{\widehat{V}_{M-1}}\right) \int_{\pi_{M-1}^{-1}\left(y_{\widehat{V}_{M-1}}\right) \cap B} d \mathscr{H}_{x}^{\operatorname{dim} V_{M}}\left(y_{\widehat{V}_{M}}\right) .}(17)
\end{gathered}
$$

Подчеркнем, что в правой части первые $M-1$ интегралов берутся по $\pi_{1}(B), \pi_{2} \circ$ $\pi_{1}^{-1}\left(y_{\widehat{V}_{1}}\right), \ldots, \pi_{M-1} \circ \pi_{M-2}^{-1} \circ \ldots \circ \pi_{1}^{-1}\left(y_{\widehat{V}_{1}}\right)$, где $y_{\widehat{V}_{1}} \in \pi_{1}(B)$. Напомним, что $\widehat{M}=$ $M$.

При проекции на горизонтальное подпространство получаем множество $\left(\Pi_{1} \cap B_{1}\right)$, где

$$
B_{1}=\left\{\left(y_{1}, \ldots, y_{\operatorname{dim} \widehat{V}_{1}}\right): \sum_{j=1}^{\operatorname{dim} \widehat{V}_{1}^{+}} y_{j}^{2}-\sum_{k=1}^{\operatorname{dim} \widehat{V}_{1}^{-}} y_{\operatorname{dim} \widehat{V}_{1}^{+}+k}^{2} \leq r^{2}\right\},
$$

а $\Pi_{1}-\operatorname{dim} V_{1}$-мерная плоскость

$$
\Pi_{1}=\left\{\left(y_{1}, \ldots, y_{\operatorname{dim} V_{1}},(\widehat{D} \varphi)_{\widetilde{V}_{1}, V_{1}}(x)\left\langle y_{1}, \ldots, y_{\operatorname{dim} V_{1}}\right\rangle\right):\left(y_{1}, \ldots, y_{\operatorname{dim} V_{1}}\right) \in \mathbb{R}^{\operatorname{dim} V_{1}}\right\} .
$$


Его $\mathscr{H}_{x}^{\operatorname{dim} V_{1}}$-мера в силу аналогичного результата из [30] равна

$$
\frac{r^{\operatorname{dim} V_{1}} \omega_{\operatorname{dim} V_{1}} \sqrt{\operatorname{det}\left(E_{\operatorname{dim} V_{1}}+(\widehat{D} \varphi)_{\widetilde{V}_{1}, V_{1}}(x)^{*}(\widehat{D} \varphi)_{\widetilde{V}_{1}, V_{1}}(x)\right)}}{\sqrt{\operatorname{det}\left(E_{\operatorname{dim} V_{1}}+(\widehat{D} \varphi)_{\widetilde{V}_{1}, V_{1}}^{+}(x)^{*}(\widehat{D} \varphi)_{\widetilde{V}_{1}, V_{1}}^{+}(x)-(\widehat{D} \varphi)_{\widetilde{V}_{1}, V_{1}}(x)^{*}(\widehat{D} \varphi)_{\widetilde{V}_{1}, V_{1}}^{-}(x)\right)}} .
$$

Так как далее последовательно применяем формулу коплощади для отображения проекции на каждое $\widehat{V}_{k}, k=2, \ldots, \widehat{M}-1$, сначала исследуем блок (13) с наибольшим номером:

$$
\left(\begin{array}{cccccc}
* & * & * & \cdots & * & E_{\operatorname{dim} V_{M}} \\
* & * & * & \cdots & * & (\widehat{D} \varphi)_{\widetilde{V}_{M}, V_{M}}(x)
\end{array}\right) .
$$

Представим $N$-мерный вектор $y$ в виде $\left(y_{V \backslash V_{M}}, y_{V_{M}}\right)$, где $y_{V_{M}}=\left(y_{N-\operatorname{dim} V_{M+1}}\right.$, $\left.\ldots, y_{N}\right)$. (Здесь и далее, где требуется, мы предполагаем, что строка транспонируется. Знак $(\cdot)^{T}$ не будем использовать, чтобы не загромождать текст.) Тогда (18) действует на $y$ как

$$
\left(y_{V_{M}}+\xi_{1}\left(y_{V \backslash V_{M}}\right), \widehat{D}_{V_{M}}(x)\left\langle y_{V_{M}}\right\rangle+\xi_{2}\left(y_{V \backslash V_{M}}\right)\right),
$$

где $\widehat{D}_{V_{M}}(x)=(\widehat{D} \varphi)_{\widetilde{V}_{M}, V_{M}}^{-}(x)=(\widehat{D} \varphi)_{\widetilde{V}_{M}, V_{M}}(x)$, а вектор-функции $\xi_{1}$ и $\xi_{2}$ - полиномы однородной степени $M$ от переменных $y_{1}, \ldots, y_{N-\operatorname{dim} V_{M}}$. Далее аргументы основываются на идее из [30]. Положим $z_{V_{M}}=y_{V_{M}}+\xi_{1}\left(y_{V \backslash V_{M}}\right)$. Тогда образ отображения, задаваемого матрицей (18), совпадает с множеством

$$
\left\{\left(z_{V_{M}}, \widehat{D}_{V_{M}}(x)\left\langle z_{V_{M}}\right\rangle+\xi\left(y_{V \backslash V_{M}}\right)\right): z_{V_{M}} \in \mathbb{R}^{\operatorname{dim} V_{M}}\right\}
$$

где $\xi\left(y_{V \backslash V_{M}}\right)=-\widehat{D}_{V_{M}}(x)\left\langle\xi_{1}\left(y_{V \backslash V_{M}}\right)\right\rangle+\xi_{2}\left(y_{V \backslash V_{M}}\right)$. Так как точки множества (19) принадлежат сублоренцеву шару, имеем

$$
\left\langle z_{V_{M}}, z_{V_{M}}\right\rangle-\left\langle\widehat{D}_{V_{M}}(x)\left\langle z_{V_{M}}\right\rangle+\xi\left(y_{V \backslash V_{M}}\right), \widehat{D}_{V_{M}}(x)\left\langle z_{V_{M}}\right\rangle+\xi\left(y_{V \backslash V_{M}}\right)\right\rangle \leq r^{2 M} .
$$

Сделаем еще одну замену переменных:

$$
z_{V_{M}} \mapsto t_{V_{M}}=z_{V_{M}}-\left(E_{\operatorname{dim} V_{M}}-\widehat{D}_{V_{M}}(x)^{*} \widehat{D}_{V_{M}}(x)\right)^{-1} \widehat{D}_{V_{M}}(x)^{*}\left\langle\xi\left(y_{V \backslash V_{M}}\right)\right\rangle .
$$

С учетом соотношения $E_{\operatorname{dim} V_{M}}-\widehat{D}_{V_{M}}(x)^{*} \widehat{D}_{V_{M}}(x)=\left(E_{\operatorname{dim} V_{M}}-\widehat{D}_{V_{M}}(x)^{*} \widehat{D}_{V_{M}}(x)\right)^{*}$ получаем, что (20) представимо в виде

$$
\begin{aligned}
& \left\langle t_{V_{M}},\left(E_{\operatorname{dim} V_{M}}-\widehat{D}_{V_{M}}(x)^{*} \widehat{D}_{V_{M}}(x)\right)\left\langle t_{V_{M}}\right\rangle\right\rangle \leq r^{2 M}+\left\langle\xi\left(y_{V \backslash V_{M}}\right), \xi\left(y_{V \backslash V_{M}}\right)\right\rangle \\
& +\left\langle\left(E_{\operatorname{dim} V_{M}}-\widehat{D}_{V_{M}}(x)^{*} \widehat{D}_{V_{M}}(x)\right)^{-1} \widehat{D}_{V_{M}}(x)^{*}\left\langle\xi\left(y_{V \backslash V_{M}}\right)\right\rangle, \widehat{D}_{V_{M}}(x)^{*}\left\langle\xi\left(y_{V \backslash V_{M}}\right)\right\rangle\right\rangle .
\end{aligned}
$$

Действительно, это следует из того, что при такой замене

$$
\left\langle t_{V_{M}}, z_{V_{M}}^{0}\right\rangle=\left\langle t_{V_{M}}, \widehat{D}_{V_{M}}(x)^{*} \widehat{D}_{V_{M}}(x)\left\langle z_{V_{M}}^{0}\right\rangle+\widehat{D}_{V_{M}}(x)^{*}\left\langle\xi\left(y_{V \backslash V_{M}}\right)\right\rangle\right\rangle
$$

для всякого $t_{V_{M}}$, где

$$
z_{V_{M}}^{0}=\left(E_{\operatorname{dim} V_{M}}-\widehat{D}_{V_{M}}(x)^{*} \widehat{D}_{V_{M}}(x)\right)^{-1} \widehat{D}_{V_{M}}(x)^{*}\left\langle\xi\left(y_{V \backslash V_{M}}\right)\right\rangle .
$$

Кроме того,

$$
\begin{array}{r}
\left\langle\xi\left(y_{V \backslash V_{M}}\right), \xi\left(y_{V \backslash V_{M}}\right)\right\rangle+\left\langle\left(E_{\operatorname{dim} V_{M}}-\widehat{D}_{V_{M}}(x)^{*} \widehat{D}_{V_{M}}(x)\right)^{-1} \widehat{D}_{V_{M}}(x)^{*}\left\langle\xi\left(y_{V \backslash V_{M}}\right)\right\rangle,\right. \\
\left.\widehat{D}_{V_{M}}(x)^{*}\left\langle\xi\left(y_{V \backslash V_{M}}\right)\right\rangle\right\rangle \geq 0 .
\end{array}
$$


Так как в силу предположения 2.12 квадратичная форма

$$
t_{V_{M}} \mapsto\left\langle t_{V_{M}},\left(E_{\operatorname{dim} V_{M}}-\widehat{D}_{V_{M}}(x)^{*} \widehat{D}_{V_{M}}(x)\right)\left\langle t_{V_{M}}\right\rangle\right\rangle
$$

положительно определена, множество

$$
\begin{aligned}
& S_{M}=\left\{t_{V_{M}}:\left\langle t_{V_{M}},\left(E_{\operatorname{dim} V_{M}}-\widehat{D}_{V_{M}}(x)^{*} \widehat{D}_{V_{M}}(x)\right)\left\langle t_{V_{M}}\right\rangle\right\rangle \leq r^{2 M}+\kappa_{M}\left(y_{V \backslash V_{M}}\right)\right\} \\
& \text { - эллипсоид и его } \mathscr{H}_{x}^{\operatorname{dim} V_{M}} \text {-мера равна }
\end{aligned}
$$

$$
\frac{\omega_{\operatorname{dim} V_{M}}\left(\sqrt{r^{2 M}+\kappa_{M}\left(y_{V \backslash V_{M}}\right)}\right)^{\operatorname{dim} V_{M}}}{\sqrt{\operatorname{det}\left(E_{\operatorname{dim} V_{M}}-\widehat{D}_{V_{M}}(x)^{*} \widehat{D}_{V_{M}}(x)\right)}},
$$

где

$$
\begin{aligned}
& \kappa_{M}\left(y_{V \backslash V_{M}}\right)=\left\langle\xi\left(y_{V \backslash V_{M}}\right), \xi\left(y_{V \backslash V_{M}}\right)\right\rangle \\
& +\left\langle\left(E_{\operatorname{dim} V_{M}}-\widehat{D}_{V_{M}}(x)^{*} \widehat{D}_{V_{M}}(x)\right)^{-1} \widehat{D}_{V_{M}}(x)^{*}\left\langle\xi\left(y_{V \backslash V_{M}}\right)\right\rangle, \widehat{D}_{V_{M}}(x)^{*}\left\langle\xi\left(y_{V \backslash V_{M}}\right)\right\rangle\right\rangle .
\end{aligned}
$$

Тогда мера образа отображения (18) совпадает с мерой образа множества (21) при отображении $t_{V_{M}} \mapsto\left(t_{V_{M}}, \widehat{D}_{V_{M}}(x)\left\langle t_{V_{M}}\right\rangle\right)$, и она будет равна

$$
\begin{aligned}
& \omega_{\operatorname{dim} V_{M}}\left(\sqrt{r^{2 M}+\kappa_{M}\left(y_{V \backslash V_{M}}\right)}\right) \operatorname{dim} V_{M} \frac{\sqrt{\operatorname{det}\left(E_{\operatorname{dim} V_{M}}+\widehat{D}_{V_{M}}(x)^{*} \widehat{D}_{V_{M}}(x)\right)}}{\sqrt{\operatorname{det}\left(E_{\operatorname{dim} V_{M}}-\widehat{D}_{V_{M}}(x)^{*} \widehat{D}_{V_{M}}(x)\right)}} \\
&=\omega_{\operatorname{dim} V_{M}}\left(\sqrt{\left.r^{2 M}+\kappa_{M}\left(y_{V \backslash V_{M}}\right)\right)^{\operatorname{dim} V_{M}}}\right. \\
& \times \frac{\sqrt{\operatorname{det}\left(E_{\operatorname{dim} V_{M}}+(\widehat{D} \varphi)_{\widetilde{V}_{M}, V_{M}}(x)^{*}\left(\widehat{D}_{\varphi}\right)_{\widetilde{V}_{M}, V_{M}}(x)\right)}}{\sqrt{\operatorname{det}\left(E_{\operatorname{dim} V_{M}}-\left(\widehat{D}_{\varphi}\right)_{\widetilde{V}_{M}, V_{M}}(x)^{*}\left(\widehat{D}_{\varphi}\right)_{\widetilde{V}_{M}, V_{M}}(x)\right)}} .
\end{aligned}
$$

Подчеркнем, что $\kappa_{M}\left(y_{V \backslash V_{M}}\right)$ - однородный полином степени $2 M$ от координат $\left(y_{1}, \ldots, y_{N-\operatorname{dim}} V_{M}\right)$. Таким образом, получаем величину

$$
\omega_{\operatorname{dim} V_{M}} \cdot \frac{\sqrt{\operatorname{det}\left(E_{\operatorname{dim} V_{M}}+(\widehat{D} \varphi)_{\widetilde{V}_{M}, V_{M}}(x)^{*}(\widehat{D} \varphi)_{\widetilde{V}_{M}, V_{M}}(x)\right)}}{\sqrt{\operatorname{det}\left(E_{\operatorname{dim} V_{M}}-(\widehat{D} \varphi)_{\widetilde{V}_{M}, V_{M}}(x)^{*}(\widehat{D} \varphi)_{\widetilde{V}_{M}, V_{M}}(x)\right)}},
$$

умноженную на

$$
\begin{aligned}
& \int_{\widehat{V}_{1}} d \mathscr{H}_{x}^{\operatorname{dim} V_{1}}\left(y_{\widehat{V}_{1}}\right) \cdots \int_{\widehat{V}_{M-1}} d \mathscr{H}_{x}^{\operatorname{dim} V_{M-1}}\left(y_{\widehat{V}_{M-1}}\right) \int_{\pi_{M-1}^{-1}\left(y_{\widehat{V}_{M-1}}\right) \cap B} d \mathscr{H}_{x}^{\operatorname{dim} V_{M}}\left(y_{\widehat{V}_{M}}\right) \\
& =\int_{D_{P} \varphi_{\Gamma}(x)\left\langle A_{1}\right\rangle} \cdots \int_{D_{P} \varphi_{\Gamma}(x)\left\langle A_{M-1}\right\rangle}\left(\sqrt{r^{2 M}+\kappa_{M}\left(y_{V \backslash V_{M}}\right)}\right)^{\operatorname{dim} V_{M}} \\
& \times d \mathscr{H}_{x}^{\operatorname{dim} V_{M-1}}\left(y_{\widehat{V}_{M-1}}\right) \ldots d \mathscr{H}_{x}^{\operatorname{dim} V_{1}}\left(y_{\widehat{V}_{1}}\right),
\end{aligned}
$$

где $A_{1}, \ldots, A_{M-1}$ - прообразы множеств из соотношения (17), лежащие в $V$, $A_{i} \subset \operatorname{span}\left\{V_{1}, \ldots, V_{i}\right\}$, которые удовлетворяют соотношениям вида (20), записанных для $V_{i}, i=1, \ldots, M-1$. Подчеркнем, что в каждом интеграле в правой части интегрирование идет только по последним $\operatorname{dim} V_{i}$ переменным (в том смысле, что образ $\widehat{D}_{P} \varphi_{\Gamma}(x)$ является $N$-мерным), $i=1, \ldots, M-1$. Из описания множества $\Pi_{1} \cap B_{1}$ видно, что для $i \leq \operatorname{dim} V_{1}$ справедливо $\left|y_{i}\right| \leq$ 
$O(r)$. Из соотношений $(20),(21)$ и дальнейших рассуждений, проведенных для $y_{\operatorname{dim} V_{1}+1}, \ldots, y_{\operatorname{dim} V_{1}+\operatorname{dim} V_{2}}$, получаем, что $\left|y_{i}\right| \leq O\left(r^{2}\right)$ для $i=\operatorname{dim} V_{1}+$ $1, \ldots, \operatorname{dim} V_{1}+\operatorname{dim} V_{2}$. Повторяя эти же рассуждения для бо́льших степеней, выводим, что $\left|y_{j}\right| \leq O\left(r^{\operatorname{deg} X_{j}}\right), j=1, \ldots, M$. Кроме того, множество $D_{P} \varphi_{\Gamma}(x)\left\langle A_{i}\right\rangle$ совпадает с образом

$$
\left(\begin{array}{c}
E_{\operatorname{dim} V_{i}} \\
(\widehat{D} \varphi)_{\widetilde{V}_{i}, V_{i}}^{-}(x)
\end{array}\right)\left\langle S_{i}\right\rangle,
$$

где $S_{i}$ - множества вида $(21)$ с заменой $M$ на $i$, в которых $\kappa_{i}$ зависит от координат с номерами от 1 до $n_{i-1}, i=M-1, \ldots, 2$, а $A_{1}=S_{1}$. Далее, по формуле площади для $D_{P} \varphi_{\Gamma}(x)$, ограниченного на множество $S_{M-1}$, имеем

$$
\begin{gathered}
\int_{D_{P} \varphi_{\Gamma}(x)\left\langle A_{M-1}\right\rangle}\left(\sqrt{r^{2 M}+\kappa_{M}\left(y_{V \backslash V_{M}}\right)}\right)^{\operatorname{dim} V_{M}} d \mathscr{H}^{\operatorname{dim} V_{M-1}} \\
=\sqrt{\operatorname{det}\left(E_{\operatorname{dim} V_{M}}+(\widehat{D} \varphi)_{\widetilde{V}_{M-1}, V_{M-1}}(x)^{*(\widehat{D} \varphi)_{V_{M-1}}, V_{M-1}}(x)\right)} \\
\times \int_{S_{M-1}}\left(\sqrt{r^{2 M}+\kappa_{M}\left(y_{V \backslash V_{M}}\right)}\right)^{\operatorname{dim} V_{M}} d \mathscr{H}^{\operatorname{dim} V_{M-1}},
\end{gathered}
$$

где $\mathscr{H}_{x}^{\operatorname{dim} V_{M-1}}$-мера $S_{M-1}$ равна

$$
\frac{\omega_{\operatorname{dim} V_{M-1}}\left(\sqrt{r^{2 M-1}+\kappa_{M-1}\left(y_{V \backslash\left(V_{M} \cup V_{M-1}\right)}\right)}\right)}{\sqrt{\operatorname{det}\left(E_{\operatorname{dim} V_{M-1}}-\widehat{D}_{V_{M-1}}(x)^{*} \widehat{D}_{V_{M-1}}(x)\right)}},
$$

a $y_{V \backslash\left(V_{M} \cup V_{M-1}\right)}$ состоит из координат с номерами от 1 до $n_{M-2}$. Аналогичные рассуждения повторим для $M-2, \ldots, 1$.

Окончательно $\mathscr{H}_{x}^{N}$-мера пересечения образа полиномиального субриманова дифференциала $\widehat{D}_{P} \varphi_{\Gamma}(x)\langle\mathscr{U}\rangle$ и сублоренцева шара ${ }^{x} \operatorname{Box}_{2}^{\mathfrak{d}}\left(\varphi_{\Gamma}(x), r\right), x \in \mathscr{U} \Subset$ $\Omega$, в координатах первого рода относительно $\varphi_{\Gamma}(x)$ равна

$$
\begin{gathered}
\frac{\sqrt{\operatorname{det}\left(E_{\operatorname{dim} V_{1}}+\left(\widehat{D}_{\varphi}\right)_{\widetilde{V}_{1}, V_{1}}(x)^{*}(\widehat{D} \varphi)_{\widetilde{V}_{1}, V_{1}}(x)\right)}}{\sqrt{\operatorname{det}\left(E_{\operatorname{dim} V_{1}}+\left(\widehat{D} \varphi^{+}\right)_{\widetilde{V}_{1}, V_{1}}^{*}(x)\left(\widehat{D} \varphi^{+}\right)_{\widetilde{V}_{1}, V_{1}}(x)-\left(\widehat{D} \varphi^{-}\right)_{\widetilde{V}_{1}, V_{1}}^{*}(x)\left(\widehat{D}^{-}\right)_{\widetilde{V}_{1}, V_{1}}(x)\right)}} \\
\times \prod_{k=2}^{M} \frac{\sqrt{\operatorname{det}\left(E_{\operatorname{dim} V_{k}}+(\widehat{D} \varphi)_{\widetilde{V}_{k}, V_{k}}(x)^{*}\left(\widehat{D}_{\varphi}\right)_{\widetilde{V}_{k}, V_{k}}(x)\right)}}{\sqrt{\operatorname{det}\left(E_{\operatorname{dim} V_{k}}-(\widehat{D} \varphi)_{\widetilde{V}_{k}, V_{k}}(x)^{*}\left(\widehat{D}_{\varphi}\right)_{\widetilde{V}_{k}, V_{k}}(x)\right)}} \cdot \mathfrak{b}(x, r, \nu),
\end{gathered}
$$

где

$$
\begin{aligned}
& \mathfrak{b}(x, r, \nu)=\int_{S_{1}(x)} \cdots \int_{S_{M-1}(x)}\left(\sqrt{r^{2 M}+\kappa_{M}\left(y_{V \backslash V_{M}}\right)}\right)^{\operatorname{dim} V_{M}} \\
& \times d \mathscr{H}_{x}^{\operatorname{dim} V_{M-1}}\left(y_{V_{M-1}}\right) \ldots d \mathscr{H}_{x}^{\operatorname{dim} V_{1}}\left(y_{V_{1}}\right) \\
& \times \omega_{\operatorname{dim} V_{M}} \prod_{j=2}^{M-1} \sqrt{\operatorname{det}\left(E_{\operatorname{dim} V_{j}}-\left(\widehat{D} \varphi^{-}\right)_{\widetilde{V}_{j}, V_{j}}^{*}(x)\left(\widehat{D} \varphi^{-}\right)_{\widetilde{V}_{j}, V_{j}}(x)\right)} \\
& \times \sqrt{\operatorname{det}\left(E_{\operatorname{dim} V_{1}}+\left(\widehat{D} \varphi^{+}\right)_{\widetilde{V}_{1}, V_{1}}^{*}(x)\left(\widehat{D} \varphi^{+}\right)_{\widetilde{V}_{1}, V_{1}}(x)-\left(\widehat{D} \varphi^{-}\right)_{\widetilde{V}_{1}, V_{1}}^{*}(x)\left(\widehat{D} \varphi^{-}\right)_{\widetilde{V}_{1}, V_{1}}(x)\right)} .
\end{aligned}
$$


Для задания меры $S L \mathscr{H}_{\mathfrak{b}}^{\nu}$ заменим в определении 2.10 соответствующий каждому шару покрытия ${ }^{x_{i}} \operatorname{Box}_{2}^{\mathfrak{d}}\left(\varphi_{\Gamma}\left(x_{i}\right), r_{i}\right)$ множитель $\prod_{j=1}^{M} \omega_{\operatorname{dim} V_{j}} r_{i}^{\nu}$ величиной $\mathfrak{b}\left(x_{i}, r_{i}, \nu\right)$, при этом множества $S_{1}, \ldots, S_{M-1}$ также будут зависеть от центра $x_{i}$, $i \in \mathbb{N}$. Дальнейшие шаги повторяют почти дословно схему из [30] с очевидными изменениями. Теорема доказана.

ЗАмЕчАниЕ 2.25. Если $\kappa_{M} \equiv \ldots \equiv \kappa_{2} \equiv 0$, то $\mathfrak{b}(x, r, \nu)=\prod_{j=1}^{M} \omega_{\operatorname{dim} V_{j}} r^{\nu}$.

ЗАмечАниЕ 2.26. В случае, когда $\operatorname{dim} V_{2}, \ldots, \operatorname{dim} V_{M}$ четные, имеем

$$
\mathfrak{b}(x, r, \nu)=\prod_{j=1}^{M} \omega_{\operatorname{dim} V_{j}} r^{\nu}+\int_{S_{1}(x)} \sum_{\substack{\beta, \mu>0,|\beta+\mu|=\nu}} K_{\beta, \mu}(x) \cdot\left(y_{V_{1}}\right)^{\beta} r^{\mu} d \mathscr{H}_{x}^{\operatorname{dim} V_{1}}\left(y_{1}\right),
$$

где $y_{V_{1}}=\left(y_{1}, \ldots, y_{\operatorname{dim} V_{1}}\right)$, а $K_{\beta, \mu}(x)$ - константы, зависящие от $x$.

\section{ЛИТЕРАТУРА}

1. Миклюков В. М., Клячин А. А., Клячин В. А. Максимальные поверхности в пространстве-времени Минковского. [Электронный ресурс]: http://www.uchimsya.info/maxsurf.pdf. 2011.

2. Naber G. L. The geometry of Minkowski spacetime. An introduction to the mathematics of the special theory of relativity. Berlin: Springer-Verl., 1992. (Appl. Math. Sci.; V. 92).

3. Nielsen B. Minimal immersion, Einstein's equations and Mach's principle// J. Geom. Phys. 1987. V. 4. P. 1-20.

4. Берестовский В. Н., Гичев В. М. Метризованные левоинвариантные порядки на топологических группах // Алгебра и анализ. 1999. Т. 11, № 4. С. 1-34.

5. Grochowski M. Reachable sets for the Heisenberg sub-Lorentzian structure on $\mathbb{R}^{3}$. An estimate for the distance function // J. Dyn. Control Syst. 2006. V. 12, N 2. P. 145-160.

6. Grochowski M. Properties of reachable sets in the sub-Lorentzian geometry // J. Geom. Phys. 2009. V. 59, N 7. P. 885-900.

7. Grochowski M. Normal forms and reachable sets for analytic Martinet sub-Lorentzian structures of Hamiltonian type // J. Dyn. Control Syst. 2011. V. 17, N 1. P. 49-75.

8. Grochowski $M$. Reachable sets for contact sub-Lorentzian metrics on $\mathbb{R}^{3}$. Application to control affine systems with the scalar input // J. Math. Sci. 2011. V. 177, N 3. P. 383-394.

9. Grochowski $M$. The structure of reachable sets for affine control systems induced by generalized Martinet sub-Lorentzian metrics // ESAIM Control Optim. Calc. Var. 2012. V. 18, N 4. P. $1150-1177$.

10. Grochowski $M$. The structure of reachable sets and geometric optimality of singular trajectories for certain affine control systems in $\mathbb{R}^{3}$. The sub-Lorentzian approach // J. Dyn. Control Syst. 2014. V. 20, N 1. P. 59-89.

11. Grochowski M. Geodesics in the sub-Lorentzian geometry // Bull. Polish Acad. Sci. Math. 2002. V. 50, N 2. P. 161-178.

12. Grochowski M. Remarks on the global sub-Lorentzian geometry // Anal. Math. Phys. 2013. V. 3, N 4. P. 295-309.

13. Korolko A., Markina I. Nonholonomic Lorentzian geometry on some H-type groups // J. Geom. Anal. 2009. V. 19, N 4. P. 864-889.

14. Korolko A., Markina I. Geodesics on H-type quaternion groups with sub-Lorentzian metric and their physical interpretation // Complex Anal. Oper. Theory. 2010. V. 4, N 3. P. 589-618.

15. Крым В. Р., Петров Н. Н. Уравнения движения заряженной частицы в пятимерной модели общей теории относительности с неголономным четырехмерным пространством скоростей // Вестн. С.-Петерб. ун-та. Сер. 1. 2007. № 1. С. 62-70.

16. Крым В. Р., Петров Н. Н. Тензор кривизны и уравнения Эйнштейна для четырехмерного неголономного распределения // Вестн. С.-Петерб. ун-та. Сер. 1. 2008. № 3. С. 68-80.

17. Craig $W$., Weinstein $S$. On determinism and well-posedness in multiple time dimensions // Proc. R. Soc. A. 2008. V. 465, N 2110. P. 3023-3046. 
18. Bars I., Terning J. Extra dimensions in space and time. New York, NY: Springer-Verl., 2010.

19. Velev M. Relativistic mechanics in multiple time dimensions // Physics Essays. 2012. V. 25, N 3. P. 403-438.

20. Карманова М. Б. Формула площади графиков на 4-мерных 2-ступенчатых сублоренцевых структурах // Сиб. мат. журн. 2015. Т. 56, № 5. С. 1068-1091.

21. Карманова М. Б. Площадь графиков на четырехмерных двухступенчатых сублоренцевых структурах // Докл. АН. 2015. Т. 463, № 4. С. 387-390.

22. Карманова М. Б. Максимальные поверхности-графики на 4-мерных 2-ступенчатых сублоренцевых структурах // Сиб. мат. журн. 2016. Т. 57, № 2. С. 350-363.

23. Карманова М. Б. Поверхности-графики на пятимерных сублоренцевых структурах // Сиб. мат. журн. 2017. Т. 58, № 1. С. 122-142.

24. Карманова М. Б. Площадь графиков на пятимерных сублоренцевых структурах // Докл. AH. 2016. Т. 467, № 6. С. 634-637.

25. Карманова М. Б. Максимальные поверхности на пятимерных групповых структурах // Сиб. мат. журн. 2018. Т. 59, № 3. С. 561-579.

26. Карманова М. Б. Вариации отображений с неголономным образом и применения к теории максимальных поверхностей // Докл. АН. 2016. Т. 468, № 3. С. 257-260.

27. Карманова М. Б. Площадь поверхностей на двуступенчатых сублоренцевых структурах с многомерным временем // Докл. АН. 2017. Т. 474, № 2. С. 151-154.

28. Карманова М. Б. Класс максимальных поверхностей-графиков на многомерных двуступенчатых сублоренцевых структурах // Докл. АН. 2018. Т. 480, № 1. С. 16-20.

29. Карманова М. Б. Графики липшицевых отображений на двуступенчатых сублоренцевых структурах с многомерным временем // Докл. АН. 2018. Т. 481, № 5. С. 474-477.

30. Карманова М. Б. Двуступенчатые сублоренцевы структуры и поверхности-графики // Изв. РАН. Сер. мат. 2020. Т. 84, № 1. С. 60-104.

31. Карманова М. Б. Достаточные условия максимальности поверхностей на двуступенчатых сублоренцевых структурах // Докл. АН. 2019. Т. 485, № 6. С. 662-666.

32. Карманова М. Б. Площадь поверхностей-графиков на группах Карно с сублоренцевой структурой // Докл. АН. 2019. Т. 485, № 2. С. 145-148.

33. Карманова М. Б. Графики негладких контактных отображений на группах Карно с сублоренцевой структурой // Докл. АН. 2019. Т. 486, № 3. С. 275-279.

34. Folland G. B., Stein E. M. Hardy spaces on homogeneous groups. Princeton: Princeton Univ. Press, 1982.

35. Pansu P. Métriques de Carnot-Carathéodory et quasi-isométries des espaces symétriques de rang un // Ann. Math. 1989. V. 129, N . P. 1-60.

36. Карманова М. Б. О полиномиальной субримановой дифференцируемости некоторых гёльдеровых отображений групп Карно // Сиб. мат. журн. 2017. Т. 58, № 2. С. 305-332.

37. Карманова М. Б. Графики липшицевых функций и минимальные поверхности на группах Карно // Сиб. мат. журн. 2012. Т. 53, № 4. С. 839-861.

38. Vodopyanov S. Geometry of Carnot-Carathéodory spaces and differentiability of mappings // The interaction of analysis and geometry. Providence, RI: Amer. Math. Soc., 2007. (Contemporary Math. V. 424). P. 247-301.

39. Ostrowsky A. Sur la détermination des bornes inférieures pour une classe des déterminants // Bull. Sci. Math. 1937. V. 61. P. 19-32.

40. Карманова М. Б. Формула площади для липшицевых отображений пространств Карно Каратеодори // Изв. РАН. Сер. мат. 2014. Т. 78, № 3. С. 53-78.

Поступила в редакиию 24 января 2019 г.

После доработки 13 апреля 2020 г.

Принята к публикации 17 июня 2020 г.

Карманова Мария Борисовна

Новосибирский государственный университет,

ул. Пирогова, 1, Новосибирск 630090

maryka@math.nsc.ru, maryka84@gmail.com 